\title{
Lateral Hypothalamic GABAergic neurons encode and potentiate
} sucrose's palatability

3 Aketzali Garcia, Alam Coss, Jorge Luis-Islas, Liliana Puron-Sierra, Monica Luna, Miguel

4 Villavicencio, Ranier Gutierrez*

5 Laboratory of Neurobiology of Appetite; Department of Pharmacology, CINVESTAV, 07360.

6 Mexico City, Mexico.

7 * Correspondence:

8 Ranier Gutierrez

9 ranier@cinvestav.mx

10 ORCID: https://orcid.org/0000-0002-9688-0289

11 Keywords: LHA $^{\text {Vgat+ }}$ neurons, feeding circuits, palatability, sucrose

12 This PDF file includes:

13 Number of pages: 45

14 Number of figures: 12 


\section{Abstract}

28 Sucrose is attractive to most species in the animal kingdom, not only because it induces a sweet taste sensation but also for its positive palatability (i.e., oromotor responses elicited by increasing sucrose concentrations). Although palatability is such an important sensory attribute, it is currently unknown which cell types encode and modulate sucrose's palatability. Studies in mice have shown that activation of GABAergic LHA ${ }^{\text {Vgat+ }}$ neurons evokes voracious eating; however, it is not known whether these neurons would be driving consumption by increasing palatability. Using optrode recordings, we measured sucrose's palatability while VGAT-ChR2 transgenic mice performed a brief access sucrose test. We found a subpopulation of $\mathrm{LHA}^{\mathrm{Vgat}+}$ neurons encodes palatability by increasing (or decreasing) their activity as a function of the increment in licking responses evoked by sucrose concentrations. Optogenetic gain of function experiments, where mice were able to choose among available water, 3\%, or $18 \%$ sucrose solution, uncovered that opto-stimulation of $\mathrm{LHA}^{\mathrm{Vgat}+}$ neurons consistently promoted higher intake of the most palatable stimulus (18\% sucrose). In contrast, if they self-stimulated near the less palatable stimulus, some VGAT-ChR2 mice preferred water over $18 \%$ sucrose. Unexpectedly, activation of $\mathrm{LHA}^{\mathrm{Vgat+}}$ neurons increased quinine intake but only during water deprivation, since in sated animals, they failed to promote quinine intake or tolerate an aversive stimulus. Conversely, these neurons promoted overconsumption of sucrose when it was the nearest stimulus. Also, experiments with solid foods further confirmed that these neurons increased food interaction time with the most palatable food available. We conclude that $\mathrm{LHA}^{\mathrm{Vgat}+}$ neurons increase the drive to consume, but it is potentiated by the palatability and proximity of the tastant. 


\section{Introduction}

60

The Lateral Hypothalamic Area (LHA) has been regarded as the "feeding center" since its lesion results in hypophagia and subsequent death (Anand and Brobeck, 1951; Teitelbaum and Epstein, 1962). It is part of a neural circuit related to feeding and reward (Delgado and Anand, 1953; Olds and Milner, 1954) as rats are willing to press a lever to deliver electrical intra-cranial self-stimulation (ICSs), and if food is available, it also promotes feeding (Delgado and Anand, 1953; Mendelson, 1967; Mogenson and Stevenson, 1967; Coons and Cruce, 1968). Moreover, if a sweet tastant is available, the rate of electrical ICSs is further increased, whereas bitter compounds decreased them (Phillips and Mogenson, 1968; Poschel, 1968), suggesting an interaction between ICSs and taste palatability. In this regard, and because of its connections with different cortical and subcortical gustatory regions (Simerly, 2004; Berthoud and Münzberg, 2011), the LHA is anatomically located to receive, process, and broadcast taste palatability information (Ferssiwi et al., 1987; Berridge and Valenstein, 1991). Pioneering electrophysiological studies have recorded gustatory-evoked responses in the LHA (Schwartzbaum, 1988; Yamamoto et al., 1989). One recent and elegant study uncovered two functional populations of LHA neurons: one activated by palatable tastants, e.g., sucrose, and another by aversive tastants, like quinine (Li et al., 2013). However, the genetic identity of the LHA cell-type(s) involved in processing palatability-related information remains elusive, and to unveil their identity is a goal of this study.

The LHA is currently viewed as a hub of various cell-types (Stuber and Wise, 2016), grossly divided into two larger populations related to feeding: the glutamatergic $\left(\mathrm{LHA}^{\text {Vglut2+ }}\right)$ and GABAergic neurons $\left(\mathrm{LHA}^{\mathrm{Vgat}+}\right)$ (Gutierrez et al., 2020). Activation of $\mathrm{LHA}^{\mathrm{Vglut2+}}$ neurons leads to reduced food intake and is aversive (Jennings et al., 2013). In contrast, stimulation of $\mathrm{LHA}^{\mathrm{Vgat}^{+}}$neurons is rewarding and produces voracious eating of both foods with nutritional value (Jennings et al., 2015; Navarro et al., 2016) and those without calories, even gnawing behavior towards the cork, an irrelevant biological stimulus (Navarro et al., 2016). Moreover, LHA ${ }^{\text {Vgat }}{ }^{+}$neurons enhance the salience of the nearest stimulus and induce reward via stimulation of their projections to the ventral tegmental area (VTA) (Nieh et al., 2016). In addition, the evoked feeding is mediated by the modulation of terminals reaching a region adjacent to the locus coeruleus (Marino et al., 2020). On the contrary, inhibition of GABAergic LHA $^{\text {Vgat+ }}$ cell somas is aversive and stops feeding (Jennings et al., 2015; Navarro et al., 2016). However, the role of $\mathrm{LHA}^{\mathrm{Vgat}+}$ neurons in processing sucrose's palatability remains to be determined.

This study identified a new role of LHA ${ }^{\mathrm{Vgat}+}$ neurons in encoding and enhancing sucrose's oromotorpalatability responses. Sucrose's palatability was defined as the enhancement of hedonically positive oromotor responses triggered by increasing sucrose concentrations (Berridge and Grill, 1983; Spector et al., 1998; Villavicencio et al., 2018). Specifically, oromotor responses include an increase in the lick rate and bout size. Thus, it should not be confused with a conscious hedonic feeling of pleasant taste that humans experience (Grill and Berridge KC, 1985; Sclafani, 1991; Berridge and Kringelbach, 2008). We found that opto-stimulation of LHA GABAergic neurons increases the consumption of the most palatable and proximal tastant. They accomplish this by potentiating the palatability of nearby gustatory stimuli. For aversive stimuli, the effect of these neurons is different. In a single bottle test, we found that water deprivation increased the tolerance for bitter compounds and gated a window of opportunity where the activation of GABAergic neurons is sufficient to temporarily reassign the 
negative hedonic value of quinine and promote its intake. Nevertheless, in a three-option preference test, mice failed to develop a quinine preference when sucrose was available under these neurons' activation, thus, demonstrating that activation of GABAergic neurons does not merely trigger indiscriminate oromotor tongue movements; rather, the animals' evoked consummatory behavior largely depends on their internal state and on the palatability of the stimulus. Moreover, optogenetic activation of $\mathrm{LHA}^{\text {Vgat+ }}$ neurons evoked many hallmark behaviors resembling those seen in LHA electrical stimulation. In this regard, and consistent with their positive role in palatability, our results could indirectly explain why electrical ICSs are further facilitated by the presence of sweet solutions (Phillips and Mogenson, 1968; Poschel, 1968). We also found that LHA ${ }^{\text {Vgat }{ }^{+}}$neurons are the common neural substrate for both reward and feeding since after repeated self-stimulation, the more the VGATChR2 mice opto-self-stimulated, the stronger the laser-induced licking they exhibited. We conclude that a subpopulation of GABAergic LHA ${ }^{\text {Vat+ }}$ neurons combines stimulus proximity and palatabilityrelated information to enhance nearby energy-rich foods' palatability and further increase

112 consummatory behaviors.

\section{Materials and methods}

115 Animal subjects

116 We used 42 VGAT-ChR2-EYFP mice (number ID 014548; Jackson Laboratory, Sacramento, CA, 117 USA) and 25 wildtype (WT) littermates, served as controls. Mice were from both sexes between 8-16 118 weeks old, and they were individually housed in their home cages and maintained in a temperature119 controlled $\left(22 \pm 1^{\circ} \mathrm{C}\right)$ room with 12:12 h light-dark cycle. Unless otherwise mentioned, chow food 120 (PicoLab Rodent Diet 20, MO, USA) and water was available ad libitum. For experiments with water 121 restriction, after each behavioral session, mice were allowed to drink water for $1 \mathrm{~h}$ daily. All procedures 122 were performed with the approval of the CINVESTAV Animal Care and Use Committee. One session 123 per day was conducted between 11:00 a.m. to 2:00 p.m.

\section{Surgical procedures}

125 All mice were anesthetized with ketamine (100 mg/kg, i.p.) and xylazine (8 mg/kg, i.p.), and then 126 placed into a stereotaxic apparatus (Stoelting, IL, USA). Lidocaine (0.05 ml) was administered 127 subcutaneously under the head's skin as a local analgesic, ophthalmic ointment (hydrocortisone, 128 neomycin, and polymyxin-B) was applied periodically to maintain eye lubrication. The antibiotic 129 enrofloxacin $(0.4 \mathrm{ml} / \mathrm{kg})$ was injected for 3 days after surgery.

130 For experiments with opto-stimulation of $\mathrm{LHA}^{\mathrm{Vgat+}}$ cell somas, a single multimode optical fiber 200$131 \mu \mathrm{m}$ core diameter with a 0.39 NA (FT200UMT; Thorlabs, NJ, USA) was implanted unilaterally 132 targeting the LHA using the following coordinates: AP: $-1.25 \mathrm{~mm}, \mathrm{ML}: \pm 1 \mathrm{~mm}, \mathrm{DV}:-4.9 \mathrm{~mm}$, from 133 bregma. For electrophysiology recordings, a custom-made optrode was unilaterally implanted 134 counterbalanced across hemispheres (AP: $-1.25 \mathrm{~mm}, \mathrm{ML}: \pm 1 \mathrm{~mm}$, from bregma; DV: $-5.2 \mathrm{~mm}$ ventral 135 to dura). The optrode comprises an array of 16 tungsten wires formvar coated (35 $\mu$ m diameter) 
surrounding and protruding $1 \mathrm{~mm}$ the single multimode optical fiber tip. No significant differences were found between hemispheres, so data were pooled together (data not shown). All experiments began 7 days after surgery to allow recovery.

\section{Histology and immunofluorescence}

141 Mice were sacrificed by an overdose of pentobarbital $(150 \mathrm{mg} / \mathrm{kg})$ and perfused with PBS, followed 142 by 4\% paraformaldehyde. Brains were fixed overnight in 4\% paraformaldehyde and gradually replaced 143 in a gradient of concentrations until 30\% sucrose. For histology, brain slides (40 $\mu \mathrm{m})$ were cut with a 144 cryostat (Thermo Scientific HM525 NX), and images were taken with a Nikon eclipse e200 microscope 145 and a Progress Gryphax microscope camera, using a 10x objective. For immunofluorescence, free146 floating brain slides $(40 \mu \mathrm{m})$ were blocked with $1 \%$ BSA, $0.2 \%$ Triton in PBS for 30 min. They were 147 then washed with $0.2 \%$ Triton in PBS three times every $10 \mathrm{~min}$, followed by the incubation with the 148 primary antibodies: mouse anti-GAD 67 primary antibody (Millipore, Mab5406, 1:1000 dilution), and 149 rabbit anti-GFP primary antibody (Invitrogen, A11122, 1:1000 dilution). Incubation took place overnight at $4{ }^{\circ} \mathrm{C}$. The next day, brain slides were washed with $0.2 \%$ Triton in PBS three times every $10 \mathrm{~min}$, then incubated with the secondary antibodies Alexa 647 goat anti-mouse (Invitrogen, A21235, 1:500 dilution), and Alexa 488 donkey anti-rabbit (Invitrogen, A21206, 1:500 dilution) during 90 min at room temperature. Afterward, we applied DAPI to stain the nuclei. Brain slides were put on slides with a mounting medium for fluorescence (Vectashield), and images were taken with a Leica confocal microscope using a $63 \mathrm{x}$ objective.

158 Sucrose reagent-grade chemical quality (Sigma-Aldrich, Mexico City, Mexico) was used in the following concentrations: water, 3, 10, and $18 \mathrm{wt} \%$. Quinine hydrochloride dihydrate $(\mathrm{QHCl})$ reagentgrade at $0.04 \mathrm{wt} \%$ (Sigma-Aldrich, Mexico City, Mexico). Artificial Saliva contained (mM): $4 \mathrm{NaCl}$, $10 \mathrm{KCl}, 6 \mathrm{NaHCO}_{3}, 6 \mathrm{KHCO}_{3}, 0.5 \mathrm{CaCl}_{2}, 0.5 \mathrm{MgCl}_{2}, 0.24 \mathrm{~K}_{2} \mathrm{HPO}_{4}, 0.24 \mathrm{KH}_{2} \mathrm{PO}_{4}$ (Zocchi et al., 2017). We added $0.05 \mathrm{mM} \mathrm{HCl}$ to adjust to $\mathrm{pH} \mathrm{7.} \mathrm{The} \mathrm{solutions} \mathrm{were} \mathrm{dissolved} \mathrm{in} \mathrm{distilled} \mathrm{water,}$ maintained under refrigeration, and used at room temperature. We also used $20 \mathrm{mg}$ chocolate pellets (Bio-Serv, NJ, USA), Chow food (PicoLab Rodent Diet 20, MO, USA), a high-fat diet with 45\% kcal\% fat (Research Diets, NJ, USA), granulated sugar cube, and cork.

168 Neural activity was recorded using a Multichannel Acquisition Processor System (Plexon, Dallas TX, 169 USA) interfaced with Med Associates to simultaneously record behavioral events (Gutierrez et al., 170 2010). Extracellular voltage signals were first amplified by an analog head-stage (Plexon, HST/16o25171 GEN2-18P-2GP-G1), then amplified and sampled at $40 \mathrm{kHz}$. Raw signals were band-pass filtered from 
$172154 \mathrm{~Hz}$ to $8.8 \mathrm{kHz}$ and digitalized at 12 bits resolution. Only single neurons with action potentials with 173 a signal-to-noise ratio of 3:1 were analyzed (Gutierrez et al., 2010). Action potentials were isolated 174 online using a voltage-time threshold window, and three principals' components contour templates 175 algorithm. Furthermore, off-line spike sorting was performed (Plexon, offline sorter), and only single 176 units with stable waveforms across the session were included in the analyses (Gutierrez et al., 2010); 177 see Supplementary Figure 1. Also, to verify waveform stability, we correlated waveform's shapes 178 recorded in the brief access test and the optotagging session.

\section{Optogenetic stimulation}

181 A $473 \mathrm{~nm}$ laser intensity was modulated by a DPSS system (OEM laser, UT, USA). Laser pulses were 182 synchronized with behavioral events with Med Associates Inc. software and TTL signal generator 183 (Med Associates Inc., VT, USA). The patch cord's optical power was at $15 \mathrm{~mW}$, and it was measured 184 with an optical power meter (PM20A, Thorlabs, NJ, USA). However, we delivered between 10 to 12.6 $185 \mathrm{~mW}$ at the fiber optic tip, depending on each fiber's efficiency. Unless otherwise mentioned, the laser 186 was turned on by $2 \mathrm{~s}$ (at $50 \mathrm{~Hz}$ ) and $4 \mathrm{~s}$ off, with $10 \mathrm{~ms}$ pulse width and a duty cycle of $50 \%$.

189 To explore the best stimulation parameters for LHA GABAergic neurons, mice were implanted with 190 an optrode in LHA and placed in a custom-made box with 18 x 13 x $7.5 \mathrm{~cm}$. With no food available, 191 the laser was turned on at different frequencies $0,2.5,5,10,20$, and $50 \mathrm{~Hz}$ semi-randomly, while 192 animals' neural activity was recorded for $20 \mathrm{~min}$. We used a Kruskal-Wallis test to compare firing rates 193 during the baseline (from -1 to $-0.04 \mathrm{~s}$ ) against the activity during laser presentation (from 0 to $2 \mathrm{~s}$ ) 194 aligned (time $=0 \mathrm{~s}$ ) to laser onset for all frequency tested. Neurons that significantly increased their 195 firing rate during the laser stimulation were named "Activated," and neurons that decreased their activity were named "Inhibited."

We used a brief access test in water-deprived mice while the LHA activity was recorded for $30 \mathrm{~min}$. We employed a licking spout consisting of independent stainless-steel needles (20-gauge) cemented together with dental acrylic at the sipper tube's tip (Villavicencio et al., 2018). Each needle was connected to a solenoid valve (Parker, Ohio, USA) by a silicon tube. The drop $(\sim 2 \mu \mathrm{L})$ was calibrated before starting the session, using a pressurized control system. The trial structure was as follows: At the beginning of the task, the sipper was extended in lick position. To start a new trial, each mouse was required to introduce its head into the central port and then elicited a dry lick to start the reward epoch 
Inter-Trial Interval (ITI), and then re-extended in a lick position into the central port. Tastant solutions (artificial saliva, water, sucrose 3\%, and sucrose 18\%) were delivered in a semi-random order.

To identify neurons whose firing rate correlated with palatability-induced oromotor responses, we used used to detect palatability-related activity in a brief access test. Briefly, during each recording session, a palatability-index (PI) was calculated. The PI reflects the overall appetitive oromotor responses elicited by each tastant delivered in the session. It was computed by averaging the lick rate during the entire reward epoch, including all the trials per session. In mice, the PI takes values between $0-8 \mathrm{~Hz}$ for sucrose stimuli (Glendinning et al., 2005). $0 \mathrm{~Hz}$ means animals ultimately rejected a solution in all trials, whereas $8 \mathrm{~Hz}$ indicates they licked continuously during the entire reward epoch, thus reflecting a greater palatability response elicited by the tastant. Then, the firing rate was calculated for a variety of time centers (from 0.25 to $6.75 \mathrm{~s}$ with $0.5 \mathrm{~s}$ steps) and multiple window sizes (from 0.5 to $7 \mathrm{~s}, 0.5 \mathrm{~s}$ steps), such that each window was estimated as the center \pm (window size/2). We identified the windows where the mean firing rate was significantly different for at least one tastant delivered (i.e., tastants, using a Kruskal-Wallis test at an alpha of 0.05). Next, we computed Pearson's correlation coefficient ( $\mathrm{r}$; the alpha level at 0.05 ) between both the PI and the firing rate on a trial-by-trial basis. The window with the largest absolute Pearson's correlation coefficient was selected as the "bestwindow." Thus, for the "best-window" and each statistical test (i.e., Kruskal-Wallis test and Pearson's correlation coefficient), a permutation assay was used for multiple-testing correction. This analysis was accomplished by shuffling the different tastant delivered 20,000 times (using the Matlab function "shuffle"). A corrected $p$-value was obtained using the following formula $p=(\mathrm{k}+1) /(\mathrm{n}+1)$, where $\mathrm{k}$ is when a permuted distribution leads to a $p$-value smaller than the original $p$-value and $\mathrm{n}$ is the number of repetitions. Only time-windows with $p$ 's $<0.05$ in both tests (Kruskal-Wallis and Pearson maximally correlated with the oromotor responses (the lick rate) elicited by sucrose's palatability on a trial-by-trial basis. Importantly, results were qualitatively similar if we used the lick bout size rather seconds) between the last and first lick in the bout (Gutierrez et al., 2006).

To evaluate whether palatability-related neurons dynamically track the changes in lick rate across the session, we computed the lick rate in the reward epoch as a function of trial types (artificial saliva (AS), water, sucrose $3 \%$, and sucrose $18 \%$ ), divided into blocks of $10^{\text {th }}$ percentile of trials each. We verified that every block across the session has the same number of trials.

242 Once the brief access test was finished, each mouse was tested in the optotagging task. For this task, 243 we removed the licking spout from the behavioral box. Over the session (15 min), the laser was turned on (at $50 \mathrm{~Hz}$ ) during $2 \mathrm{~s}$ or $7 \mathrm{~s}$, followed by $10 \mathrm{~s}$ off. 
245 Laser activated neurons (pLHA ${ }^{\text {Vgat }}$ ) were detected by using two methods: 1) a Kruskal-Wallis to compare firing rates during a baseline (from -1 to $-0.04 \mathrm{~s}$ ) against the activity during the presentation of laser (from $0-2 \mathrm{~s}$, aligned to laser onset). 2) Cumsum statistic (Gutierrez et al., 2006) to obtain the first bin ( $1 \mathrm{~ms}$ resolution) after the first laser pulse that significantly increased the firing rate above baseline (from -20 to $0 \mathrm{~ms}$ ). Only neurons showing a significant value in both tests were considered laser-activated $\mathrm{pLHA}^{\mathrm{Vgat}}$ From the population $\mathrm{pLHA}^{\mathrm{Vgat}}$ neurons, we identified two types of populations: 1) ChR2-expressing cells $\mathrm{LHA}^{\mathrm{Vgat}+}$ or early neurons. These neurons were those with an action potential evoked within $\leq 15 \mathrm{~ms}$ latency (Buonomano, 2003), measured by a cumsum statistic; and 2) laser-activated late neurons: these were those with an action potential occurring after $>15 \mathrm{~ms}$ latency. Also, to classify non-LHA ${ }^{\text {Vgat }}$ neurons, we used Kruskal-Wallis test as described above. Neurons with no significant modulation were classified as "unmodulated."

The front panel of an operant conditioning chamber (Med Associates Inc, VT, USA) was equipped Associates Inc, VT, USA). To determine the best stimulation parameters to induce feeding behavior, a group of naïve mice had free access to sucrose $10 \%$ solution. For open loop stimulation, mice were opto-stimulated by alternating blocks of $5 \mathrm{~min}$ "off" and $5 \mathrm{~min}$ "on" across a $40 \mathrm{~min}$ session (Nieh et al., 2016). During opto-stimulated blocks, the laser was turned "on" regardless of the mice's behavior and spatial position in the chamber. A different opto-stimulation frequency $(0,2.5,5,10,20$, and 50 $\mathrm{Hz}$ ) was delivered daily. The laser bound feeding index was measured as the number of licks in a 2.5 $\mathrm{s}$ window from laser onset divided by total licks in the session, hereafter named laser bound feeding, and it was plotted as a function of laser frequency.

\section{Pre-stimulation task}

A group of naïve mice was placed in an operant conditioning chamber with a central port. A door blocked the access to the sipper during the first $15 \mathrm{~min}$ (pre-stimulation period. Each mouse was optostimulated with all pre-stimulation protocols $(0,5,10$, and $15 \mathrm{~min})$ following a Latin square design. Then, the door was opened, and a 10\% sucrose solution was available during the next 15 min. During the pre-stimulation period, the laser was turned on for $2 \mathrm{~s}$ and off for $4 \mathrm{~s}$.

277 To determine whether opto-stimulation of LHA GABAergic neurons drives the intake with a bias 278 towards the most palatable stimulus available, a new group of naïve mice was tested in an open loop 279 stimulation task (alternating 5 min no-laser and 5 min "on" ( $2 \mathrm{~s}$ on, $4 \mathrm{~s}$ off) blocks across a $40 \mathrm{~min}$ 280 session). The operant chamber was equipped with three ports, where mice had free access to water 
(central sipper), a 3\% sucrose solution (left sipper), and 18\% sucrose solution (right sipper). Lateral sippers were counterbalanced across animals. This task comprises four baseline sessions (with no photostimulation, data not shown), and eleven test sessions were pooled together. The number of licks given to the sipper filled with sucrose $18 \%$, across the eleven sessions was also plotted.

For this experiment, we video tracked the mouse's distance and head angle relative to each of the three sippers. The sippers' position and three anatomical animal points, the nose, the neck, and the tail's base, were extracted using the DeepLabCut Python package (Mathis et al., 2018; Nath et al., 2019). We define the mouse position as the coordinates ( $\mathrm{x}$ and $\mathrm{y}$ ) for the nose, whereas the head direction was a $2 \mathrm{D}$ vector going from the neck to the nose. For each video frame, each sipper's distance was calculated (in pixels), and the angle between the head direction and position of each sipper (in degrees). An angle of $0^{\circ}$ means the mouse is looking at the sipper, and $180^{\circ}$ means facing the opposite direction. For distance measuring, 100 pixels correspond to approximately $6 \mathrm{~cm}$. The mouse position and direction were extracted from the train of stimulation's first laser pulse, and the following $6 \mathrm{~s}$ window was analyzed to find out if the mice initiated a licking behavior at any of the 3 sippers. Then, we built a two-dimensional array containing the probability of licking any sipper given the mouse's distance and angle during the first laser of each opto-stimulation train.

A new group of animals was placed in the circular arena's center $(50 \mathrm{~cm}$ in diameter). Three or two food plates were equidistant to each other, and each contained either Chow, high-fat diet, or granulated sugar cube. Mice were opto-stimulated by alternating 5 min block with no-laser and 5 min laser block across the session (20 min). In open loop, the laser was turned "on" regardless of the mice's behavior and spatial position in the arena. All sessions were recorded and analyzed with bonsai software (https://open-ephys.org/bonsai). We calculated the mouse's centroid at each frame and used that information to create a heatmap of its position. The distance of the mouse's centroid from each stimulus was computed. A food interaction occurred when the distance was below $60 \mathrm{~mm}$, and the animal stayed there for at least $1 \mathrm{~s}$. Food plates were weighed at the beginning and the end of each session.

\section{Closed-loop stimulation with three options}

310 To evaluate whether activation of $\mathrm{LHA}^{\mathrm{Vgat}+}$ neurons induces preference for the most proximal stimulus, we used a closed-loop stimulation protocol (in the same mice from Open loop stimulation with three options), over eleven additional sessions (40 min each). As noted above, mice were placed in an operant conditioning chamber with three ports (water, central sipper; 3\% sucrose solution, left sipper; $18 \%$ sucrose solution, right sipper). Photostimulation was delivered when subject made a head entry in the central port (containing a sipper filled with water), laser was turned on by $2 \mathrm{~s}$ followed by a time out of $4 \mathrm{~s}$ (where the laser could not be re-activated), after this, a new laser onset ( $2 \mathrm{~s}$ “on") occurred when mice performed a new head-entry. Thus, in this experiment, the less palatable stimuli (water) was the 
318 nearest to opto-self-stimulation. During photostimulation, sucrose preference index was measured as

319 the number of licks of $18 \%$ sucrose divided by total licks for sucrose $18 \%+$ water. Values higher than

$320 \quad 0.5$ indicate sucrose preference.

321 To evaluate the laser-bound feeding development across the sessions, we correlated laser bound 322 feeding during open loop stimulation sessions vs. closed-loop stimulation for the first three and the last 323 five sessions.

325 Closed-loop in an open-field

326 In a circular arena, four stimuli were located equidistant to each other: wood cork, Chow, chocolate pellets, or a sipper filled with a 10\% sucrose solution. During the session, a homemade computer vision program tracked in real-time the position of the subject. Each mouse needs to enter the designated area to receive opto-self-stimulation ( $2 \mathrm{~s}$ laser on and $4 \mathrm{~s}$ off). Mice need to leave and re-enter the designated area to begin a new trial. Only one designated area was used per session, and it remained in the same position for up to 3 or 4 consecutive sessions. Each session's duration was $40 \mathrm{~min}$ (mice were the same as those used in the previous task).

335 For the same group of mice used in the Closed-loop with three options, and on subsequent days, the 336 central port's stimulus was replaced across twenty-eight sessions, following the protocols described in 337 Table 1.

338 Table 1. Protocol used for closed-loop stimulation with central port stimulus replaced.

Stimuli in central port

\begin{tabular}{cc}
\hline \hline Water & Last 5 sessions of the closed-loop \\
\hline Extinction (No opto-stimulation) & 4 \\
\hline Water & 3 \\
\hline Empty sipper & 3 \\
\hline Water & 3 \\
\hline 0.04\% Quinine & 4 \\
\hline Airpuff & 4 \\
\hline Unavoidable airpuff & 3 \\
\hline $\begin{array}{c}\text { Sucrose } 18 \% \text { (Water was placed } \\
\text { in the sucrose 18\% port) }\end{array}$ & 4 \\
\hline
\end{tabular}




\section{Closed-loop stimulation task with one option}

341 Mice were put in a smaller and custom-made box with 18 x 13 x $7.5 \mathrm{~cm}$ internal dimensions. The front 342 wall was equipped with one single sipper and a contact lickometer with a $\mathrm{V}$-shape to register individual licks. First, mice were tested, for 3 days, with a $0.04 \%$ quinine solution under sated state (fed mice) and then for 3 more days under a $23 \mathrm{~h}$ water-deprived condition, each session lasted 20-min. Then, mice were tested for 2 days with a sipper filled with 18\% sucrose, in a sated state, and then 2 more days with water-deprived condition. Opto-self-stimulation $(2 \mathrm{~s}$ on with a time out of $4 \mathrm{~s}$, where the laser could not be activated) was delivered when mice made a head entry in the central port. Waterdeprived mice had access to ad libitum water for $1 \mathrm{~h}$ after the session.

To evaluate whether activation of GABAergic neurons enhances oromotor-palatability responses, we performed a brief access test with a new group of mice over 31 sessions. The behavioral setup conditions were the same as described above, and as tastant solutions, we used water, sucrose 3\%, and sucrose $18 \%$ (delivered in a semi-random order). The trial structure was similar, but this time to start a new trial, each mouse was required to introduce its head into the central port to turn "on" the laser. Then, elicited a dry lick to start the reward epoch $(7 \mathrm{~s})$. Each head entry triggers the laser onset for $2 \mathrm{~s}$ "on" (or in different sessions for $7 \mathrm{~s}$ "on"; see Table 2). At the end of the session, mice had access to ad libitum water for $1 \mathrm{~h}$. Protocols are described in Table 2 below:

Table 2. Protocol for opto-self-stimulation in a brief access test.

Laser on

Time on

\# Sessions (30 min each)

\begin{tabular}{cll}
\hline \hline All trials & $2 \mathrm{~s}$ & 6 \\
\hline Water trials & $2 \mathrm{~s}$ & 3 \\
\hline Sucrose 3\% trials & $2 \mathrm{~s}$ & 3 \\
\hline Sucrose 18\% trials & $2 \mathrm{~s}$ & 3 \\
\hline All trials & $7 \mathrm{~s}$ & 4 \\
\hline Water trials & $7 \mathrm{~s}$ & 3 \\
\hline Sucrose 3\% trials & $7 \mathrm{~s}$ & 3 \\
\hline Sucrose 18\% trials & $7 \mathrm{~s}$ & 3 \\
\hline Mock laser (sucrose 3\% trials) $*$ & $7 \mathrm{~s}$ & 3 \\
\hline
\end{tabular}

*For mock laser sessions, mice were connected to a false fiber optic, whereas the real laser was glued outside the skull to emit blue light.

In addition, to verify that LHA GABAergic neurons were capable of sustained activation over $7 \mathrm{~s}$ of optostimulation. We plotted the PSTH of pLHA $^{\text {Vgat }}$, non-LHA ${ }^{\text {Vgat }}$, and unmodulated neurons using the same method previously described. 
Data Analysis

All data analysis was performed using MATLAB (The MathWorks Inc., Natick, MA) and GraphPad Prism (La Jolla, CA, USA). We used the mean \pm sem and the $\alpha$ level at 0.05 .

\section{Results}

Initially, we determined the optimal stimulation parameters to activate LHA GABAergic neurons. This was accomplished by implanting an optrode in naïve mice that constitutively expressed $\mathrm{ChR} 2$, fused the vesicular $\gamma$-aminobutyric acid transporter (hereafter referred to as VGAT-ChR2 mice) (Zhao et al., 2011) (Figures 1A-B). We found that a laser stimulation of $50 \mathrm{~Hz}$ (10 ms width) evoked the strongest LHA neuronal responses (Figures 1C-F) (Gigante et al., 2016). Unless otherwise mentioned, this frequency was used for all subsequent experiments.

\section{LHA neurons encode sucrose's oromotor palatability responses}

In rats, previous electrophysiological studies suggest that ensembles of LHA neurons process palatability-related information (Norgren, 1970; Schwartzbaum, 1988; Yamamoto et al., 1989; Li et al., 2013). Based on these results, we then asked whether LHA neurons could provide information about sucrose's palatability using a behavioral task with only palatable stimuli (i.e., artificial saliva (AS), water, sucrose $3 \mathrm{wt} \%$, and sucrose $18 \mathrm{wt} \%$ ). To this end, taste responses were recorded from a total of 284 LHA neurons, while VGAT-ChR2 mice performed a brief access test (Figure 2A). To uncover palatability-responsive neurons, we analyzed neuronal activity using a previously described "best-window analysis" (Villavicencio et al., 2018) (see Materials and Methods). This strategy detects the optimal interval, where the firing rate best correlates with the sucrose's palatability index (PI) computed as the average lick rate (or bout size), on a session by session basis, evoked by each gustatory stimulus in the Reward epoch (Figure 2B; one-way ANOVA, F ${ }_{(3,108)}=10.35, p<0.0001$; responses of two representative LHA neurons: as expected, we found one whose activity increased as the sucrose concentration increased (positive Pearson's correlation, $\mathrm{r}=0.87$; Figure $2 \mathrm{C}$ left), and the other was negatively associated with palatability oromotor responses evoked by sucrose $(\mathrm{r}=-0.64$; Figure 2C right). Figure 2D shows the normalized activity exhibiting either positive (59/284 neurons, $21 \%$ ) or negative correlations $(76 / 284,27 \%)$ with the PI. Also, qualitatively similar results were found using the bout size to compute the PI (Supplementary Figure 2B). Thus, these data confirmed that the anti-correlated with the sucrose solution's PI. 


\section{Dynamic tracking of palatability over the entire course of a behavioral session}

403 Having demonstrated that LHA neurons encode sucrose's palatability-induced oromotor responses, we 404 then explored whether LHA palatability-related neurons can dynamically adjust their responses to track the changes in sucrose's palatability across the session when the animals would approach satiation. We found that the lick rate declined across the session as a function of sucrose's palatability (Figure 2E; RM ANOVA, main effect of stimuli, $\mathrm{F}_{(3,402)}=163.7, p<0.0001$; effect of time, $\mathrm{F}_{(9,1206)}=100.4, p<$ 0.0001 ; and stimuli by time interaction, $\left.\mathrm{F}_{(27,3618)}=16.54, p<0.0001\right)$. Briefly, early in the session, animals licked more for all stimuli, but as the session progressed, the lick rate declined, especially for AS and water but not for sucrose $(3 \%$ and $18 \%)$, indicating that they tracked palatability responses rather than satiety (Figure 2E). Similar to what we have shown in the Nucleus Accumbens Shell (NAcSh) (Villavicencio et al., 2018), a brain region that sends outputs to LHA and is involved in feeding and also contains palatability-related neurons (O'Connor et al., 2015; Villavicencio et al., 2018), we also found that the LHA neuron's firing rate (either increasing or decreasing neurons) adjust across the session to reflect sucrose's palatability (Figure 2F). These data indicate that within the session, as the animals approach satiety, the responses of the Palatability related-neurons tracked the rapid decline in the lick rate mainly for the two less palatable stimuli (i.e., AS and water) but not for the higher sucrose-concentration (sucrose 3\% and 18\%), suggesting these neurons are tracking palatability and no satiety.

To identify LHA ${ }^{\text {Vgat+ }}$ neurons from our recordings, we opto-stimulated the same mice recorded in the brief access test seen in Figure 2. We verified the stability of the waveforms between tasks (i.e., brief access test and optotagging). We only included single units with stable waveforms in the analysis (see Materials and Methods; Supplementary Figure 1). Figure 3A displays the setup and the normalized (Zscore) activity of laser-activated neurons (48\%; 137/284) (for details, see Material and Methods). Since these neurons may include ChR2 expressing cells and other LHA neurons modulated by indirect polysynaptic feedback of afferent fibers from other areas in the brain (Nieh et al., 2015), we decided to name them putative $\mathrm{LHA}^{\mathrm{Vgat}}\left(\mathrm{pLHA}^{\mathrm{Vgat}}\right.$ ) neurons. Note that $\mathrm{pLHA}^{\mathrm{Vgat}}$ neurons comprise the ensemble recruited by the optogenetic stimulation of LHA GABAergic neurons, suggesting they convey similar information. Therefore, we next evaluated whether $\mathrm{pLHA}^{\mathrm{Vgat}}$ neurons were correlated with sucrose's palatability. In total, 50\% (69/137) of laser-activated neurons exhibited responses that were significantly palatability related; specifically, 34\% showed a positive correlation (46 out of 137), whereas $17 \%$ (23 out of 137) had a negative correlation with sucrose's palatability (Figure 3B; Positive vs. Negative chi-square test $(1,274)=10.25, p<0.01)$. Figure $3 \mathrm{C}$ shows the population activity of pLHA $^{\text {Vgat }}$ neurons with either a positive (yellow) or negative (purple) Pearson's correlation coefficients with the PI (red). Furthermore, from all recorded LHA neurons that encode sucrose induced oromotorpalatability responses with a Positive correlation ( $\mathrm{n}=59$, see Figure $2 \mathrm{D})$, we found that more than $78 \%$ $(46 / 59)$ belonged to the pLHA ${ }^{\text {Vgat }}$ (Figure 3D). In contrast, only 30\% (23/76) of pLHA ${ }^{\text {Vgat }}$ neurons were 
0.0001). Similar results were found when we analyzed the Laser-activated late neurons (hereafter named Late neurons) from the pLHA $^{\text {Vat }}$ population. These neurons exhibited a delayed laser evoked action potential with a slow latency $(>15 \mathrm{~ms}), \mathrm{n}=66$, see Supplementary Figure $3 \mathrm{~A}$. We compared the proportion of Late neurons against all palatability-responsive neurons recorded in the LHA and found that $41 \%$ (24/59) belonged to the Positive palatability-related population, and only $8 \%(6 / 76)$ were Negative palatability-related neurons (Supplementary Figure 3B-C; chi-square test $(1,135)=20.65, p<$ 0.0001). Thus, $\mathrm{pLHA}^{\mathrm{Vgat}}$ encode sucrose's palatability with a biased towards positive correlations.

448 To confirm the identity of $\mathrm{LHA}^{\mathrm{Vgat+}}$ neurons, we searched for neurons in which a brief pulse of lightevoked an action potential with an early latency $(\leq 15 \mathrm{~ms})$ that would reflect the expression of ChR2 in their somas (Buonomano, 2003). In total, from the $\mathrm{pLHA}^{\mathrm{Vgat}}$ population, $52 \%(71 / 137)$ neurons exhibited an early response to blue light (Figures 3E-F), suggesting that these pLHA $^{\text {Vat }}$ neurons were LHA $^{\text {Vgat+ }}$ neurons. We found that over half of the identified LHA ${ }^{\text {Vgat+ }}{ }^{+}$neurons, 55\% (39/71), were involved in encoding sucrose's oromotor-palatability, and the remaining (45\%) were unmodulated by sucrose. From the LHA neurons related to sucrose's oromotor-palatability, $31 \%$ of LHA $^{\text {Vgat }}{ }^{+}$neurons positively correlated with sucrose's palatability (22/71), and 24\% were anticorrelated (17/71) (Figure $3 \mathrm{G}$; Positive vs. Negative chi-square test $(1,142)=0.88, \mathrm{p}=0.34)$. The LHA ${ }^{\text {Vgat }}{ }^{+}$neuronal responses with positive and negative Pearson's correlation coefficients with lick-related palatability responses are seen in Figure $3 \mathrm{H}$. Once again, we compared the proportion of opto-identified LHA ${ }^{\text {Vgat }}{ }^{+}$neurons against all palatability-responsive neurons recorded in the LHA. We found a trend to encode sucrose's palatability in a positive rather than negative manner. That is, 37\% (22/59) of $\mathrm{LHA}^{\mathrm{Vgat}^{+}}$neurons belonged to the Positive palatability-related population, whereas only $22 \%(17 / 76)$ were Negative palatability-related neurons (Figure 3I; Positive vs. Negative chi-square test $(1,135)=3.59, \mathrm{p}=0.057$ ). Altogether, these data suggest that $\mathrm{LHA}^{\mathrm{Vgat}{ }^{+}}$neurons tend to encode sucrose's palatability in a positive rather than in a negative manner. More important, our data agree with the idea that the LHA ${ }^{\text {Vgat+ }}$ 2015).

\section{Non-LHA ${ }^{\text {Vgat }}$ neurons negatively encode sucrose's palatability}

In contrast, we found that $25 \%$ of LHA neurons exhibited a laser-induced inhibition (70/284, non$\mathrm{LHA}^{\text {Vgat }}$ neurons, Figure 3J, blue trace), and the remaining 27\% (77/284) were unmodulated during blue light stimulation (Figure 3J, black trace). Unlike pLHA ${ }^{\mathrm{Vgat}}$ neurons, only 7\% (5 out of 70) of the non-LHA ${ }^{\text {Vgat }}$ neurons showed a positive correlation, whereas the vast majority, 36\% (25 out of 70), had a negative correlation with sucrose's palatability (Figures 3K and 3L; Positive vs. Negative chi-square test $_{(1,140)}=16.97, p<0.0001$ ). These results suggest that non-LHA ${ }^{\text {Vat }}$ neurons (perhaps glutamatergic Vglut2 neurons) preferentially encode sucrose's palatability with negative correlations (Figure 3M; Positive vs. Negative chi-square test $(1,135)=11.46, p<0.001)$. 
If a subpopulation of LHA GABAergic neurons preferentially encodes sucrose's palatability by increasing firing rates, its optogenetic stimulation should promote increased sucrose intake. To characterize its impact on sucrose intake, we used an open loop optogenetic stimulation involving 5 min blocks with laser and no-laser stimulation, while naïve VGAT-ChR2 mice had ad lib access to a sipper filled with sucrose $10 \%$ (Figure $4 A$ ). We analyzed the intake as a function of laser frequency (Figure 4B; two-way ANOVA, frequency by blocks interaction, $\mathrm{F}(35,96)=2.268, p<0.01$ ), a post-hoc test uncovered that in the first three blocks with laser, $50 \mathrm{~Hz}$ stimulation induced a significant increase in sucrose intake (Figure 4B; Blocks 2, 4, and 6) compared with the previous blocks of no-laser (Figure representative open loop session. At $50 \mathrm{~Hz}$, we found that feeding (licking) was elicited within a 0.72 $\mathrm{s} \pm 0.03$ latency from laser onset, and it abruptly stopped $(0.27 \mathrm{~s} \pm 0.1)$ after laser offset (see blue dash rectangles). VGAT-ChR2 mice exhibited an increase in the laser-bound feeding as the laser frequency increased (Figure 4D; two-way ANOVA, group by frequency interaction, $\mathrm{F}(5,36)=12.86, p<0.0001$ ). Thus, to induce sucrose intake, $\mathrm{LHA}^{\mathrm{Vgat+}}$ neurons require continued activation. Our data then confirmed that $50 \mathrm{~Hz}$ is the best stimulation frequency to drive neuronal responses and sucrose intake.

\section{${ }_{\text {LHA }}{ }^{\text {gat }{ }^{+}}$neurons do not induce a persistent hunger state}

A previous study showed that brief optogenetic stimulation of "hunger-inducing" AgRP neurons in the arcuate nucleus before food availability promotes consummatory behavior that persists for several minutes in the absence of continued AgRP neuron activation (Burnett et al., 2016; Chen et al., 2016). Based on this study, we tested whether the pre-stimulation of $\mathrm{LHA}^{\mathrm{Vgat}}{ }^{+}$neurons could evoke a similar hunger state. Unlike AgRP neurons (Burnett et al., 2016; Chen et al., 2016), pre-stimulation of LHA $^{\text {Vgat }}{ }^{+}$neurons failed to evoke and sustain subsequent sucrose intake (see Supplementary Figure 4; two-way ANOVA, group by pre-stimulation protocol, $\left.\mathrm{F}_{(3,56)}=0.50, p=0.68\right)$. Our results suggest that feeding occurs while LHA ${ }^{\text {Vgat+ }}$ neurons are active (Figure $4 \mathrm{C}$ ), but they do not induce a persistent hunger state as AgRP neurons do (Chen et al., 2016).

To test whether activation of $\mathrm{LHA}^{\mathrm{Vgat}+}$ neurons promotes the intake of the most palatable available stimulus, naïve WT and VGAT-ChR2 mice were opto-stimulated in an open loop protocol, with access to three adjacent ports containing water, sucrose 3\%, or sucrose $18 \%$ (Figure 5A). As expected, both WT and VGAT-ChR2 mice preferred sucrose $18 \%$ over water, and sucrose 3\% (Figure 5B; WT: twoway ANOVA main effect tastants; $\mathrm{F}_{(2,1560)}=543.3, p<0.0001$; VGAT-ChR2: $\mathrm{F}_{(2,3144)}=983, p<$ 0.0001). However, we found that sated VGAT-ChR2 mice increased their intake of $18 \%$ sucrose mainly during the laser-activated blocks compared with controls (Figure 5B-right; two-way ANOVA group by laser block interaction $\left.\mathrm{F}_{(7,1568)}=31.86, p<0.0001\right)$ and rarely licked in the absence of stimulation (Figure 5C). In this regard, VGAT-ChR2 mice seem to counteract the evoked sucrose intake by voluntarily restraining consumption in no-laser blocks (Blocks 3, 5, and 7), resulting in no 
significant differences between groups in the total intake of $18 \%$ sucrose (Figure 5C, see black squares; unpaired Student's t-test, $t(196)=1.512, p=0.1320)$. A between days analysis revealed that the evoked $18 \%$ sucrose intake began from the first stimulation day (Figure 5D; RM ANOVA laser blocks, $\mathrm{F}(1,22)$ $=64.64, p<0.0001$ ), although sucrose consumption ramps up throughout the days (Figure 5D; RM ANOVA main effect days, $\left.\mathrm{F}_{(10,220)}=2.169, p<0.05\right)$. This suggests that $\mathrm{LHA}^{\mathrm{Vgat}+}$ neurons, rather than inducing hunger per se, induced a learning process that potentiates the intake of the most palatable stimulus and confines consumption mainly in the presence of opto-stimulation.

The small increase in water and sucrose 3\% intake observed during opto-stimulation of LHA ${ }^{\text {Vgat }}$ neurons could be explained by random stimulation near those tastants (Figure $5 \mathrm{~B}$ right panel). Accordingly, we found in laser blocks 2,4 , and 6 , a significant increase in water and sucrose $3 \%$ in the VGAT-ChR2 mice compared with the WT mice that completely neglected those tastants (Figure 5B, $\wedge p<0.05$ for sucrose $3 \%$; $\dagger p<0.05$ for water). We hypothesize that this additional intake can be attributed to trials where the laser-activation occurred near these less palatable stimuli. To answer this, we employed a videography analysis (Figure 6A-B). When opto-stimulation occurred in the distance minor to 50 pixels (approximately $3 \mathrm{~cm}$, see Materials and Methods) relative to sucrose 3\% or water ports, the licking probability increased significantly after opto-stimulation (Figure 6B; Sucrose 3\%: F $(9,155)=29.33, p<0.0001$; Water: one-way ANOVA; $\left.F_{(9,153)}=12.74, p<0.0001\right)$. The angle of the head was less informative (Figure 6C-D; Sucrose 3\%: $\mathrm{F}_{(17,147)}=0.71, p=0.78$; Water: one-way ANOVA; F $(17,145)=0.96, p=0.49$; Sucrose 18\%: one-way ANOVA; F $(17,153)=0.49 p=0.95)$. the lateral port with $18 \%$ sucrose, increasing their overconsumption (Figure 6C, Video 1). Moreover, we observed that for the sucrose $18 \%$ port, even when the mouse position was twice as far (i.e., $6 \mathrm{~cm}$ ) from sucrose $18 \%$ port, the licking probability increased significantly (Figure 6B right panel; one-way ANOVA, $\left.F_{(9,161)}=82.87, p<0.0001\right)$. These data are consistent with a study showing that optostimulation of LHA ${ }^{\text {Vgat+ }}$ neurons promotes the intake of the nearest stimulus (Nieh et al., 2016) but further demonstrates that the most palatable stimulus has nearly twice the distance of attraction than the other less palatable options.

Open loop activation of $\mathrm{LH}^{\mathrm{Vgat+}}$ neurons also increases the time spent and drives the intake of the most palatable solid food available

We also explored whether LHA GABA neurons could induce the intake of the most palatable solid food available. Thus, using an open loop protocol, we opto-stimulate these neurons while mice choose among different solid foods. We found that optogenetic activation of $\mathrm{LHA}^{\mathrm{Vgat}^{+}}$neurons increased the time spent near the most palatable food available. VGAT-ChR2 mice spent more time near the highfat diet than the granulated sugar cube or the Chow food pellet, relative to WT group. Also, the intake of transgenic mice was higher for high-fat diet than the other food stimuli (see Supplementary Figure

5535 and Video 2; Time spent: one-way ANOVA, $\mathrm{F}_{(5,306)}=38.52, p<0.0001$; Intake: one-way ANOVA, $\left.554 \mathrm{~F}_{(5,282)}=68.76, p<0.0001\right)$, however, both WT and VGAT-ChR2 mice consumed similar amounts of 555 high-fat diet $(p=0.0985$.), perhaps because it is a highly palatable food. When mice were able to 
choose between a sugar cube and a Chow pellet, now the activation of these neurons increased the time spent and consumption of sugar cube over Chow (see Supplementary Figure 6 and Video 3; Time spent: one-way ANOVA, F $(3,96)=16.13, p<0.0001$; Intake: one-way ANOVA, $\mathrm{F}_{(3,92)}=13.65, p<$ 0.0001). Our data suggest that open loop stimulation of LHA GABAergic neurons promotes the attraction to and the intake of the most palatable food available.

To further test the idea that $\mathrm{LHA}^{\mathrm{Vgat}+}$ neurons could induce a preference for a proximal (but less palatable) stimulus over a distal one with higher hedonic value, we used a closed-loop stimulation protocol. That is, the same mice (from Figure 5) were photostimulated, but here the laser was triggered by each head entry in the central port (i.e., opto-self-stimulation). This closed-loop configuration guarantees that stimulation only occurs proximal to water, the least palatable stimulus of the three in the box (Figure $7 A$-right). We found that activation of $\mathrm{LHA}^{\mathrm{Vgat}+}$ neurons is rewarding since transgenic mice visited the central port significantly more and performed a higher number of opto-selfstimulations than the WT mice (Figure 7B; unpaired Student's t-test, $t_{(196)}=12, p<0.0001$ ). Surprisingly, the total number of licks in the session seen in Figure 7C shows that during closed-loop stimulation, the VGAT-ChR2 mice explored and consumed more water than the WT mice (two-way ANOVA group by tastants interaction, $\mathrm{F}_{(2,585)}=13.09, p<0.0001$, post hoc test at water, $\left.\mathrm{p}<0.0001\right)$. Note that in both open loop and closed-loop protocols, the WT and VGAT-ChR2 mice exhibited, at the end of the session, a similar number of licks for $18 \mathrm{wt} \%$ sucrose (Figure 7C; two-way ANOVA group by protocol interaction $\left.\mathrm{F}_{(1,391)}=2.172, p=0.1414\right)$. That is, the total licks for sucrose $18 \%$ were not significantly different between groups, suggesting that these neurons do not interfere with the overall attractiveness of sucrose. However, by counting the number of licks given when the laser was turned on (in a $2.5 \mathrm{~s}$ window from laser onset- see Figure 7D), we uncovered a striking change in preference. As noted, in the open loop, the VGAT-ChR2 mice exhibited a higher preference for sucrose $18 \mathrm{wt} \%$, over both sucrose $3 \mathrm{wt} \%$ and water. Now, in the closed-loop protocol, the same transgenic mice consumed more water compared with WT (two-way ANOVA group by tastants interaction, $\mathrm{F}$ (2, $585)=12.18, p<0.0001$, post hoc test at water, $p<0.0001$ ), and the consumption of water was about the same as sucrose $18 \%(p=0.8731$, n.s.; Figure 7D). The increase in water intake was selective to the photostimulation window since the transgenic mice only drank sucrose $18 \%$ and neglected water when the laser was turned off (see arrow Figure 7E; two-way ANOVA group by tastants interaction, F $(2,585)=64.16, p<0.0001$, post hoc test at water vs. sucrose $18 \%, \mathrm{p}<0.0001)$. Thus, $\mathrm{LHA}^{\mathrm{Vgat}^{+}}$neurons could promote water intake, but only when it is the nearest stimulus. The sucrose preference index (Figure 7F) showed that during the open loop, all VGAT-ChR2 mice preferred sucrose $18 \%$ over water (Figure 7F; values $>0.5$ and near to 1 ), whereas in the closed-loop protocol, when water was the nearest stimulus, most mice ( $\mathrm{n}=9$ out of 12 ), significantly diminished their sucrose preference (Figure 7F, see the drop in preference index; paired Student's $t$-test, $t(58)=7.98, p<0.0001)$. From these 9 animals, 6 exhibited a higher preference for water over sucrose $18 \%$ (Preference index values < 0.5 ; Video 4). Figure $7 \mathrm{G}$ shows the color-coded location of fiber optic tips in each VGAT-ChR2 mice plotted in Figure "7F." However, the location of the optical fibers does not explain the variability in preference. 
Perhaps the variability is due to different behavioral strategies used by each mouse. Nevertheless, the large variability in the drop of sucrose preference resembles the findings with sweet-induced facilitation by electrical ICSs (Poschel, 1968). These data suggest that $\mathrm{LHA}^{\mathrm{Vat}^{+}}$neurons drive consummatory behavior by integrating the stimulus's proximity and hedonic value.

600

After repeated stimulation, the correlation between laser-bound feeding and self-stimulation strengthens

604

Previous studies have shown that after repeated LHA bulk stimulation (either electrically or with optogenetic targeting all cell-types together), subjects switched from exhibiting stimulus-bound feeding to only self-stimulating, indicating that LHA evoked feeding and reward could represent two distinct processes (Gigante et al., 2016; Urstadt and Berridge, 2020). Consequently, we explored whether laser-bound feeding changed after repeated optogenetic stimulation. In contrast to previous studies, we observed that laser-bound feeding induced by $\mathrm{LHA}^{\mathrm{Vgat}+}$ neurons strengthened across sessions (Figure 8A, First 3 days: $r=0.12, p=0.69$; Last 5 stimulation days: $r=0.63, p<0.05$ ) and after repeated stimulation, both opto-self-stimulation and laser-bound feeding exhibited a robust correlation (Figure 8B, First 3 days: $r=0.42, p=0.16$; Last 5 days: $r=0.61, p<0.05$ ), suggesting that LHA evoked-feeding (licking) involves a learning process (Sharpe et al., 2017) and that LHA and Chow pellets

617 The "proximity effect" evoked by $\mathrm{LHA}^{\mathrm{Vgat+}}$ neurons was not restricted to liquid tastants, it also applied to solid foods. In a real-time place preference arena with four stimuli (Figure 9A), when chocolate pellets or Chow food were the designated foods near opto-self-stimulation of LHA ${ }^{\text {Vgat }}{ }^{+}$neurons, mice also increased the time spent near those foods (Figure 9B; Pellet: one-way ANOVA; F $(4,145)=99.05$, $p<0.0001$; Chow, $\mathrm{F}_{(4,145)}=182, p<0.0001$ ) as well as their intake (Figure 9C; Pellet: unpaired Student's t-test, $t_{(68)}=3.234, p<0.05$; Chow: unpaired Student's t-test, $t_{(68)}=3.651, \mathrm{p}<0.05$ ), see Video 5. Thus, these LHA GABAergic neurons reinforced the approach and exploration to any, if not the most appetitive stimulus, that happened to be proximal to the opto-stimulation.

\section{LHA ${ }^{\text {Vgat }+}$ neurons activation is rewarding}

627 In the same WT and VGAT-ChR2 mice tested in the closed-loop configuration seen in Figure 7, we 628 went on to show that mice visited the central water-port to opto-self-stimulate. To do this, we performed extinction sessions with the laser disconnected (Figure 10A). We observed a rapid decrease in the number of opto-self-stimulations (Figure 10B; Extinction phase, gray shadow). As expected,

631 self-stimulation rapidly recovered when the laser was turned on again (Figure 10, see water after 
632 extinction). Thus, $\mathrm{LHA}^{\mathrm{Vgat+}}$ neurons convey a hedonically positive, rewarding signal (Jennings et al., 633 2015).

\section{LHA ${ }^{\text {Vgat+ }}$ neurons also promote licking an empty sipper}

636 A previous study demonstrated that chemogenetic activation of LHA ${ }^{\text {Vgat }}{ }^{+}$neurons increased gnawing 637 to non-edible cork (Navarro et al., 2016). In the absence of other stimuli but cork, we also observed 638 gnawing behavior in some mice, although we did not systematically study stereotypy (see Video 6). 639 Instead, we further explored this idea by replacing water, in the central port, with an empty sipper 640 (Figure 10, Empty sipper). Transgenic mice continued opto-self-stimulating at the same place as if 641 water were still present in the central port. $\mathrm{LHA}^{\mathrm{Vgat}+}$ stimulation evoked licking an empty sipper 642 compared with WT mice (Figure 10C, unpaired Student's t-test, $t_{(40)}=2.532, p<0.05$ ). Thus, LHA $^{\text {Vgat }^{+}}$ 643 neurons evoke an imminent urge to express consummatory behavior even in the form of dry licking an 644 empty sipper, a non-biological relevant stimulus, but only when it is the nearest stimulus to 645 photostimulation. This effect is perhaps also mediated by an increase in the rewarding value of 646 appetitive oromotor responses per se.

When sucrose is available, activation of $\mathrm{LH}^{\mathrm{Vgat}+}$ neurons neither promotes liquid intake of an aversive bitter tastant nor tolerance of punishment, but it further increases sucrose consumption

650 To explore whether the "stimulus-proximity effect" was also applied to aversive stimuli, we replaced the central stimulus (water) with aversive stimuli, including quinine (a tastant that humans experience as bitter taste) or airpuffs (Figure 10A). Upon quinine presentation, the number of licks given in the central port sharply decreased (Figure 10C, green), but transgenic mice continued to self-stimulate and go to the lateral port to lick for sucrose $18 \%$ (Figure 10D, green). We obtained similar results to those in quinine stimulation during the airpuff delivery phase (Figure 10D, dark gray). Next, we required the animal to lick, at least once, the central sipper to trigger opto-stimulation (thus making the airpuffs unavoidable). Under this condition, transgenic mice completely stopped self-stimulation (Figure 10B, cyan) and aborted 18\% sucrose intake from the lateral port (Figure 10D, cyan). These results suggest that, in sated mice, $\mathrm{LHA}^{\mathrm{Vgat}+}$ neurons did not induce quinine intake nor increase tolerance to airpuffs.

661 Finally, we explored whether proximity to the most palatable tastant further facilitated its 662 overconsumption. Thus, we exchanged the position of water and sucrose $18 \%$. When sucrose $18 \%$ was 663 delivered in the central port, transgenic mice greatly overconsumed it (Figure 10C, pink). The intake 664 of sucrose $18 \%$ was higher than for all the other tastants tested previously $(p<0.0001)$. These results 665 collectively suggest that activation of $\mathrm{LHA}^{\mathrm{Vgat}+}$ neurons is rewarding and promotes increased 666 consumption of the nearest stimulus even if it is not the most palatable (e.g., water and empty sipper). 
667 If the nearest stimulus happens to be the most palatable (i.e., sucrose 18\%), then LHA GABA neurons 668 further facilitated its consumption.

\section{LHA GABA neurons increased quinine intake but only in water-deprived mice}

671

672

673

674

675

676

677

678

679

680

681

682

Having demonstrated that in the presence of sucrose, activation of these neurons failed to increase quinine intake (Figure 10C green), with a new group of naïve mice, we then explored whether LHA ${ }^{\text {Vgat+ }}$ neurons could induce quinine intake when it was the only option. As expected, in sated mice, we found that activation of $\mathrm{LHA}^{\mathrm{Vgat}+}$ neurons did not affect quinine intake compared with WT (Figure 11A; unpaired Student's t-test, $\mathrm{t}(46)=0.9925, \mathrm{p}=0.3262)$. Surprisingly, it promoted a higher quinine intake when transgenic mice were water-deprived (Figure 11B; unpaired Student's t-test, t (46) =2.958, p < 0.01), specifically during photostimulation window (Figure 11B; unpaired Student's t-test, t $(46)=$ $4.473, \mathrm{p}<0.0001$ ), suggesting that activation of these neurons is sufficient to increase the acceptance of bitter tastants but only during water deprivation. In contrast, we found that regardless of homeostatic needs, activation of these neurons increased sucrose $18 \%$ intake relative to WT mice (Figure 11C-D; unpaired Student's t-test, t $(30)=6.933, \mathrm{p}<0.0001$; unpaired Student's t-test, $\mathrm{t}(30)=3.183, \mathrm{p}<0.01)$.

\section{Activation of $\mathrm{LHA}^{\mathrm{Vgat+}}$ neurons enhances palatability}

We next explored whether, at equal stimulus distance, these neurons could enhance palatability responses. To do this, in a new group of water-deprived mice, we employed a brief access test again. In this task, we delivered gustatory stimuli water, sucrose $3 \%$, and sucrose $18 \%$ from the same sipper tube, but in different trials. Figure $12 A$ displays the trial's structure (same conventions as in Figure 2). We found that both groups increased their licking rate as a function of sucrose's concentration, reflecting its palatability (Figure 12B; two-way ANOVA main effect of tastants, $\mathrm{F}_{(2,174)}=9.101, p<$ 0.001). However, during the $2 \mathrm{~s}$ of laser stimulation, the VGAT-ChR2 mice exhibited a greater lick rate for all three tastants. But, when the laser was turned "off," these mice abruptly stopped licking for water and sucrose 3\% compared with the WT group (Figure 12B, right panel; two-way ANOVA group by tastants interaction, $\left.\mathrm{F}_{(2,174)}=5.293 p<0.01\right)$. Moreover, when stimulation was paired with water trials, transgenic mice selectively increased their lick rate to water relative to WT (Figure 12C, blue line, and arrow; two-way ANOVA group by tastants interaction, $\left.\mathrm{F}_{(2,84)}=6.009, p<0.01\right)$, even surpassing licking responses evoked by the most palatable sucrose $18 \%$. A similar enhancement of oromotor responses was observed by pairing sucrose 3\% trials with LHA $^{\text {Vgat+ }}$ opto-self-stimulation (Figure 12D, orange; two-way ANOVA group by tastants interaction, $\mathrm{F}_{(2,84)}=16.72, p<0.0001$ ). Likewise, the laser-bound feeding (licking rate) was strongest when sucrose $18 \%$ was paired with the optogenetic stimulation (Figure 12E, red, see arrow; two-way ANOVA group by tastants interaction, $\left.\mathrm{F}_{(2,84)}=16.49, p<0.0001\right)$.

Finally, we show that palatability responses could be artificially extended as long as LHA ${ }^{\text {Vgat+ }}{ }^{\text {neurons }}$ were continuously activated. For this, we photostimulated them for up to $7 \mathrm{~s}$. We observed a similar 
704

705

706

707

708

709

710

711

712

713

714

715

716

717

718

719

720

721

722

723

724

725

726

727

728

729

730

731

732

733

734

735

736

737

738

739

740

741

742

enhancement pattern, maintained for the duration of opto-stimulation (see Figure 12F-I see arrows; All trials: two-way ANOVA main effect of tastants, $\mathrm{F}_{(2,114)}=12.53, p<0.0001$; Water trials: two-way ANOVA group by tastants interaction, $\mathrm{F}_{(2,84)}=9.850, p<0.001$; Sucrose 3\% trials : two-way ANOVA group by tastants interaction, $\mathrm{F}_{(2,84)}=33.47, p<0.0001$; Sucrose $18 \%$ trials : two-way ANOVA group by tastants interaction, $\left.\mathrm{F}_{(2,84)}=8.360, p<0.001\right)$, suggesting that $\mathrm{LHA}^{\mathrm{Vgat}+}$ neurons can adjust the enhancement of oromotor palatability responses by simply sustaining its neuronal activity. We verified that using optrode recordings, $7 \mathrm{~s}$ optogenetic stimulation produced sustained LHA $^{\text {Vgat }}{ }^{+}$neuronal responses (Figure 12J). Moreover, transgenic mice did not merely use the light as a cue to guide behavior since laser-stimulation with a mock optical fiber failed to increase licking (Figure 12K; twoway ANOVA group by tastants interaction, $\left.\mathrm{F}_{(2,54)}=1.619, p=0.207\right)$. In sum, our data demonstrate that activation of $\mathrm{LHA}^{\mathrm{Vgat+}}$ neurons triggers a reinforcing signal that amplifies the positive hedonic value of proximal stimuli, promoting overconsumption.

\section{Discussion}

LHA has historically been viewed as a critical center for feeding (Anand and Brobeck, 1951; Delgado and Anand, 1953; Teitelbaum and Epstein, 1962), although it also processes sucrose's palatability related information (Norgren, 1970; Ono et al., 1986; Li et al., 2013). In addition to caloric value, sucrose's palatability is the affective or hedonic attribute of sweetness that determines whether to like it or not (Grill and Berridge KC, 1985). Despite the importance of palatability to promote overconsumption, the specific LHA cell-type(s) identity involved in processing sucrose's palatability has remained elusive. Our results demonstrated that a subpopulation of $\mathrm{LHA}^{\text {Vgat+ }}{ }^{+}$GABAergic neurons encodes sucrose's palatability by exhibiting two opposite modulatory patterns, either correlating positively or negatively with the palatability index, with a bias towards a positive correlation. Furthermore, opto-stimulation of $\mathrm{LHA}^{\mathrm{Vgat}+}$ cell somas promoted the approach and intake of the most salient available and palatable tastant. In contrast, opto-self-stimulation promoted increased liquid intake of the less-attractive and proximal stimuli, despite having more palatable but distal tastants available. These findings show that $\mathrm{LHA}^{\mathrm{Vgat}+}$ neurons compute and/or combine, at least, two types of information: one related to stimulus proximity and the other to palatability that results in enhancing stimulus-saliency (Nieh et al., 2016). Experiments with solid food also unveiled that transgenic mice spent more time near the most palatable food available. More important, among the many other functions already ascribed to these neurons (see below and Nieh et al., 2016), our data uncovered a new function of $\mathrm{LHA}^{\mathrm{Vgat+}}$ neurons as physiological potentiators of sucrose-induced oromotor palatability responses.

Previous studies have shown that $\mathrm{LHA}^{\mathrm{Vgat}+}$ population contains many subpopulations with different functional responses, with at least one ensemble responding to appetitive (approach) and others to consummatory behaviors (Jennings et al., 2015). Furthermore, activation of these neurons is rewarding, induces voracious eating (Jennings et al., 2015; Navarro et al., 2016), and promotes interaction of the nearest stimulus (either objects or other mice) (Nieh et al., 2016), suggesting that they play a role in multiple motivated behaviors. However, it is also known that LHA connects and receives direct inputs 
from multiple cortical and subcortical gustatory regions (Simerly, 2004; Berthoud and Münzberg, 2011), and some electrophysiological studies report that LHA neurons respond to gustatory stimuli in particular to tastant palatability (Norgren, 1970; Ono et al., 1986; Schwartzbaum, 1988; Yamamoto et al., 1989; Karádi et al., 1992). As noted in rodents, palatability is operationally defined as the enhancement of hedonically positive oromotor responses induced by stimulating the tongue with ascending sucrose concentrations (Berridge and Grill, 1983; Spector et al., 1998; Villavicencio et al., 2018). Specifically, these hedonically positive oromotor responses may include an increase in the lick rate or the bout size. In agreement with this definition, we found that a subpopulation of LHA palatability related-neurons tracked licking oromotor-related responses by increasing or decreasing their activity in a sucrose-concentration dependent manner. Within a session, LHA neurons tracked the sucrose's palatability rather than satiety or hunger signals. We showed that these LHA ${ }^{\text {Vgat+ }}$ neurons could function as enhancers of sucrose's palatability. Optogenetic activation of these neurons can also enhance water's palatability if it is the nearest stimulus. We found an increased lick rate for water during these neurons' activation as if the animal were sampling a high sucrose concentration. Moreover, their activation promotes the intake of liquid sucrose (or solid granulated sugar cube). These neurons also increased the consumption of other more palatable stimuli like high-fat pellets (see Supplementary Figure 5; Video 2), similar to the other GABAergic neurons but in zona incerta (Zhang and van den Pol, 2017). Thus, our data demonstrate that activation of LHA GABAergic drives the intake of the most palatable stimulus available in the animal's environment.

Although activation of $\mathrm{LHA}^{\mathrm{Vgat+}}$ promotes substantial feeding behavior, it has become clear that LHA $^{\text {Vgat+ }}$ neurons are not directly involved in evoking hunger (Burnett et al., 2016; Navarro et al., 2016; Marino et al., 2020), as AgRP neurons in the arcuate do (Chen et al., 2016). In this regard, and unlike AgRP neurons, pre-stimulation of LHA GABAergic neurons did not trigger a sustained sucrose intake in the absence of continuous activation (Supplementary Figure 4). Thus, to induce a consummatory behavior, these neurons are required to remain active. Moreover, and in agreement with these findings, we found that the intake induced by $\mathrm{LHA}^{\mathrm{Vgat}+}$ neurons convey a positive valence signal that combines both stimulus proximity and palatability related information. Thus, these neurons enhance the saliency of nearby hedonically positive stimuli, whether those stimuli are sapid chemicals, as we show, or social cues, as in the approach behavior towards juvenile or female intruders and new objects (Nieh et al., 2016).

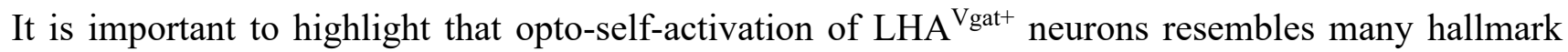
behaviors evoked by LHA electrical stimulation. In particular, our results could shed some light on why, at low-intensity electrical currents, lever pressing to deliver ICSs only occurs if food (or sucrose) is in close proximity (Mendelson, 1967; Coons and Cruce, 1968; Valenstein et al., 1968; Valenstein and Phillips, 1970). Consistent with its role in enhancing sucrose's palatability, a subpopulation of GABAergic neurons could indirectly explain why sweet tastants further potentiate the rate of LHA electrical ICSs (Poschel, 1968). We concluded that a subpopulation of LHA ${ }^{\text {Vat }{ }^{+}}$neurons could account for many, if not all, of these electrically induced phenomena. Also, we found differences between unspecific LHA stimulation and our targeted LHA ${ }^{\mathrm{Vgat}^{+}}$stimulation. Unlike electrical LHA stimulation, 4-12). In contrast, to the high variability found in rats exhibiting LHA electrically-induced feeding, 
one study reported that only 12 of 34 rats showed stimulus-bound feeding (Valenstein and Cox, 1970). cell-types found in LHA, simultaneously (Urstadt and Berridge, 2020). These studies reported that after repeated LHA stimulation (either electrically or with optogenetics), some subjects switched from exhibiting stimulus-bound feeding to only self-stimulating, suggesting that these two processes were flexible and not correlated (Gigante et al., 2016; Urstadt and Berridge, 2020). In contrast, we found that repeated stimulation of $\mathrm{LHA}^{\mathrm{Vgat}+}$ neurons increases laser-induced feeding (licking). Likewise, the correlation between optogenetic self-stimulation and laser-bound feeding increases through stimulation days (Figure 8). That is, the more the animals self-stimulated, the stronger the evoked laser-bound licking was. Thus, $\mathrm{LHA}^{\mathrm{Vgat+}}$ neurons are the common neural substrate for evoking both feeding and reward. Though, it was recently shown that they do it by using two projection pathways; reward via a VTA projection and feeding via the peri-Locus Coeruleus nuclei (Marino et al., 2020).

796

Given that LHA is involved in reward and aversion (Ono et al., 1986), we next tested whether LHA ${ }^{\text {Vgat+ }}$ neurons could promote bitter tastants' intake. We found that opto-stimulation of $\mathrm{LHA}^{\mathrm{Vgat}^{+}}$neurons failed to promote quinine intake, a bitter tastant, or tolerance of an aversive airpuff when sucrose was also available. Thus, these neurons play a minimal role in increasing the preference for a proximal but aversive stimulus over distal sucrose. Furthermore, in sated mice and using a single bottle test, these neurons also failed to increase quinine intake. Unexpectedly, during water deprivation, a copious quinine consumption was observed. These results demonstrate that the consummatory drive induced by the activation of GABAergic neurons largely depends on the palatability of stimulus and animal's internal state. These results agree with previous findings that chemogenetic inhibition of LHA ${ }^{\text {Vat }^{+}}$ neurons did not alter the quinine rejection responses. Thus, in sated mice, these neurons are not necessary to express hedonically negative responses induced by bitter tastants (Fu et al., 2019). However, they did not explore their sufficiency. Our results showed that water deprivation temporarily gated LHA ${ }^{\text {Vgat+ }}$ neurons to promote quinine intake and further demonstrated that their activation is sufficient to increase acceptance of an aversive tastant during water deprivation.

The LHA comprises multiple heterogeneous and overlapping populations based on the expression of genetic markers for neuropeptides, receptors, and proteins involved in the synthesis and vesicular packaging of neurotransmitters (Bonnavion et al., 2016; Mickelsen et al., 2019). Thus, the LHA ${ }^{\text {Vat }^{+}}$

813 population can be further subdivided into GABA neurons expressing leptin receptor (LepRb)

814 (Leinninger et al., 2009; Mickelsen et al., 2019), or the neuropeptide galanin (Gal), or neurotensin (Nts)

815 (Qualls-Creekmore et al., 2017; Kurt et al., 2019; Mickelsen et al., 2019). It is known that the activation 816 of LHA-GABA-LepRb is rewarding (Giardino et al., 2018), similar to LHA ${ }^{\text {Vat }^{+}}$neurons. Likewise, 817 LHA GABA-Gal expressing neurons are related to food reward behavior, but unlike LHA ${ }^{\text {Vgat }}$, these

818 neurons do not promote food consumption (Qualls-Creekmore et al., 2017). From these 819 subpopulations, only the LHA GABA-Nts neurons recapitulate some (but not all) of the behavioral 820 effects reported here. Unlike LHA ${ }^{\text {Vgat }+}$ neurons, a previous study found that LHA GABA-Nts neurons do not increase chow intake. Instead, they promote the liquid intake of palatable tastants (water, $\mathrm{NaCl}$, and sucrose). In sated mice, chemogenetic activation of GABA-Nts increased the intake of bitter quinine, albeit with lower magnitude, when it was the only liquid available to eat with chow food, a marked contrast to our findings with broad LHA GABAergic neurons activation. Although they did 
825

826

827

828

829

830

831

832

833

834

835

836

837

838

839

840

841

842

843

844

845

846

847

848

849

850

851

852

853

854

855

856

857

858

859

860

not explore quinine intake in the absence of chow food or water-deprived mice. However, they performed a two-bottle test and found that GABA-Nts neurons increased water intake over quinine, suggesting that activation of these neurons is not involved in driving mice's preference for bitter tastants, similar to what we found for the $\mathrm{LHA}^{\mathrm{Vgat}+}$ population. Also, similar to our findings, activation of LHA GABA-Nts induced water-drinking, which was further facilitated if the solution was sucrose (Kurt et al., 2019). Thus, it will be interesting to determine the role that LHA GABA-Nts neurons play in encoding and potentiating sucrose's palatability. It follows that the LHA contains nested functions encoded in each subpopulation (or cell-types) that are then recruited selectively to exert a more refined control over feeding and reward.

A caveat of this study is that we employed bacterial artificial chromosomes (BAC) transgenic strain mouse, VGAT-ChR2, that constitutively expressed ChR2 in GABAergic neurons expressing the gene for the VGAT (Zhao et al., 2011). In this model, we cannot rule out the unintended activation of GABAergic terminals from distal regions (Thoeni et al., 2020), which also occurs with classic electrical stimulation. Nevertheless, in the more specific transgenic model, the Vgat-ires-Cre mice have found a similar feeding-bound behavior for chow food (Marino et al., 2020), as we have shown here (see Chow in Figures 9B and C; and Video 5). Moreover, the VGAT-ChR2 transgenic model affords important advantages such as a consistent expression of ChR2 (Zeng and Madisen, 2012) and heritable transgene expression patterns across experimental cohorts (Ting and Feng, 2013), which increased reproducibility across animals tested. It is also a more selective model to characterize GABAergic neurons (excluding the glutamatergic component) and their effects recapitulating classical effects observed with electrical LHA stimulation (Delgado and Anand, 1953; Phillips and Mogenson, 1968).

In summary, here we found that at least a subpopulation of $\mathrm{LHA}^{\mathrm{Vgat+}}$ neurons could be an important hub that links stimulus-proximity and palatability related information to potentiate the palatability of nearby energy-rich foods, especially those containing sucrose.

\section{Conflict of interests}

The authors declare no competing financial interests.

\section{Author Contributions}

A.G., A.C., J. LI., and R.G. designed research; A.G., A.C., J. LI., L.PS., and M.L. performed research; M.V. contributed unpublished analytic tools; A.G., A.C., J. LI., and R.G. analyzed data; A.G. and R.G. wrote the paper; all authors reviewed and approved the manuscript.

\section{Funding}

This project was supported in part by Productos Medix 3247, Cátedra Marcos Moshinky, fundación Miguel Alemán Valdés, CONACyT Grants Fronteras de la Ciencia 63 and 10862, and Problemas Nacionales 464 ( to R.G.). 


\section{Acknowledgments}

862 Aketzali Garcia had a CONACyT doctoral fellowship, and data in this work is part of her doctoral dissertation in the Posgrado en Ciencias Biomédicas of the Universidad Nacional Autónoma de México. We want to thank Martin Vignovich and Professor Sidney A. Simon for helpful comments in an early version of this manuscript. We thank Mario Gil Moreno for building homemade optrode and valuable help of Juan de Dios Rodriguez-Callejas during the acquisition of confocal microscope images. We also thank Ricardo Gaxiola, Victor Manuel García Gómez, and Fabiola Hernandez Olvera for invaluable animal care.

This manuscript has been released as a pre-print at Biorxiv, (Garcia et al., 2020).

\section{References}

872
Anand, B. K., and Brobeck, J. R. (1951). Localization of a "feeding center" in the hypothalamus of the rat. Proceedings of the Society for Experimental Biology and Medicine. Society for Experimental Biology and Medicine 77, 323-4.

Berridge, K. C., and Grill, H. J. (1983). Alternating ingestive and aversive consummatory responses suggest a two-dimensional analysis of palatability in rats. Behavioral neuroscience 97, 563-73.

Berridge, K. C., and Kringelbach, M. L. (2008). Affective neuroscience of pleasure: reward in humans and animals. Psychopharmacology (Berl) 199, 457-480. doi:10.1007/s00213-008-1099-6.

Berridge, K. C., and Valenstein, E. S. (1991). What psychological process mediates feeding evoked by electrical stimulation of the lateral hypothalamus? Behav. Neurosci. 105, 3-14. doi:10.1037//07357044.105.1.3.

Berthoud, H.-R., and Münzberg, H. (2011). The lateral hypothalamus as integrator of metabolic and environmental needs: from electrical self-stimulation to opto-genetics. Physiol. Behav. 104, 29-39. doi:10.1016/j.physbeh.2011.04.051.

Bonnavion, P., Mickelsen, L. E., Fujita, A., de Lecea, L., and Jackson, A. C. (2016). Hubs and spokes of the lateral hypothalamus: cell types, circuits and behaviour. J. Physiol. (Lond.) 594, 6443-6462. doi:10.1113/JP271946.

Buonomano, D. V. (2003). Timing of neural responses in cortical organotypic slices. Proc. Natl. Acad. Sci. U.S.A. 100, 4897-4902. doi:10.1073/pnas.0736909100.

Burnett, C. J., Li, C., Webber, E., Tsaousidou, E., Xue, S. Y., Brüning, J. C., et al. (2016). Hunger-Driven Motivational State Competition. Neuron 92, 187-201. doi:10.1016/j.neuron.2016.08.032.

Chen, Y., Lin, Y.-C., Zimmerman, C. A., Essner, R. A., and Knight, Z. A. (2016). Hunger neurons drive feeding through a sustained, positive reinforcement signal. Elife 5. doi:10.7554/eLife.18640.

Coons, E. E., and Cruce, J. A. (1968). Lateral hypothalamus: food current intensity in maintaining selfstimulation of hunger. Science 159, 1117-9. doi:10.1126/science.159.3819.1117. 
896

897

898

899

900

901

902

903

904

905

906

907

908

909

910

911

912

913

914

915

916

917

918

919

920

921

922

923

924

925

926

927

928

929

930

931

932

933

934

935

Delgado, J. M., and Anand, B. K. (1953). Increase of food intake induced by electrical stimulation of the lateral hypothalamus. The American journal of physiology 172, 162-8. doi:10.1152/ajplegacy.1952.172.1.162.

Ferssiwi, A., Cardo, B., and Velley, L. (1987). Gustatory preference-aversion thresholds are increased by ibotenic acid lesion of the lateral hypothalamus in the rat. Brain Res. 437, 142-150. doi:10.1016/00068993(87)91535-6.

Fu, O., Iwai, Y., Narukawa, M., Ishikawa, A. W., Ishii, K. K., Murata, K., et al. (2019). Hypothalamic neuronal circuits regulating hunger-induced taste modification. Nat Commun 10, 1-14. doi:10.1038/s41467-019-12478-x.

Garcia, A., Coss, A., Luis-Islas, J., Puron-Sierra, L., Luna, M., Villavicencio, M., et al. (2020). Lateral Hypothalamic GABAergic neurons encode and potentiate sucrose's palatability. bioRxiv, 2020.06.30.180646. doi:10.1101/2020.06.30.180646.

Giardino, W. J., Eban-Rothschild, A., Christoffel, D. J., Li, S.-B., Malenka, R. C., and de Lecea, L. (2018). Parallel circuits from the bed nuclei of stria terminalis to the lateral hypothalamus drive opposing emotional states. Nat. Neurosci. 21, 1084-1095. doi:10.1038/s41593-018-0198-x.

Gigante, E. D., Benaliouad, F., Zamora-Olivencia, V., and Wise, R. A. (2016). Optogenetic Activation of a Lateral Hypothalamic-Ventral Tegmental Drive-Reward Pathway. PLoS ONE 11, e 0158885. doi:10.1371/journal.pone.0158885.

Glendinning, J. I., Chyou, S., Lin, I., Onishi, M., Patel, P., and Zheng, K. H. (2005). Initial licking responses of mice to sweeteners: effects of tas1r3 polymorphisms. Chem. Senses 30, 601-614. doi:10.1093/chemse/bji054.

Grill, and Berridge KC (1985). "Taste reactivity as a measure of the neural control of palatability.," in Progress in Psychobiology and Physiological Psychology. (Orlando, FL.: Academic Press), 1-54.

Gutierrez, R., Carmena, J. M., Nicolelis, M. A. L., and Simon, S. A. (2006). Orbitofrontal ensemble activity monitors licking and distinguishes among natural rewards. J. Neurophysiol. 95, 119-133. doi:10.1152/jn.00467.2005.

Gutierrez, R., Fonseca, E., and Simon, S. A. (2020). The neuroscience of sugars in taste, gut-reward, feeding circuits, and obesity. Cell. Mol. Life Sci. doi:10.1007/s00018-020-03458-2.

Gutierrez, R., Simon, S. A., and Nicolelis, M. A. (2010). Licking-induced synchrony in the taste-reward circuit improves cue discrimination during learning. The Journal of neuroscience : the official journal of the Society for Neuroscience 30, 287-303. doi:10.1523/JNEUROSCI.0855-09.2010.

Jennings, J. H., Rizzi, G., Stamatakis, A. M., Ung, R. L., and Stuber, G. D. (2013). The inhibitory circuit architecture of the lateral hypothalamus orchestrates feeding. Science 341, 1517-1521. doi:10.1126/science. 1241812 .

Jennings, J. H., Ung, R. L., Resendez, S. L., Stamatakis, A. M., Taylor, J. G., Huang, J., et al. (2015). Visualizing hypothalamic network dynamics for appetitive and consummatory behaviors. Cell 160, 516-27. doi:10.1016/j.cell.2014.12.026.

Karádi, Z., Oomura, Y., Nishino, H., Scott, T. R., Lénárd, L., and Aou, S. (1992). Responses of lateral hypothalamic glucose-sensitive and glucose-insensitive neurons to chemical stimuli in behaving rhesus monkeys. J. Neurophysiol. 67, 389-400. doi:10.1152/jn.1992.67.2.389. 
936

937

938

939

940

941

942

943

944

945

946

947

948

949

950

951

952

953

954

955

956

957

958

959

960

961

962

963

964

965

966

967

968

969

970

971

972

973

974

975

Kurt, G., Woodworth, H. L., Fowler, S., Bugescu, R., and Leinninger, G. M. (2019). Activation of lateral hypothalamic area neurotensin-expressing neurons promotes drinking. Neuropharmacology 154, 1321. doi:10.1016/j.neuropharm.2018.09.038.

Leinninger, G. M., Jo, Y.-H., Leshan, R. L., Louis, G. W., Yang, H., Barrera, J. G., et al. (2009). Leptin acts via leptin receptor-expressing lateral hypothalamic neurons to modulate the mesolimbic dopamine system and suppress feeding. Cell Metab. 10, 89-98. doi:10.1016/j.cmet.2009.06.011.

Li, J. X., Yoshida, T., Monk, K. J., and Katz, D. B. (2013). Lateral hypothalamus contains two types of palatability-related taste responses with distinct dynamics. The Journal of neuroscience : the official journal of the Society for Neuroscience 33, 9462-73. doi:10.1523/JNEUROSCI.3935-12.2013.

Marino, R. A. M., McDevitt, R. A., Gantz, S. C., Shen, H., Pignatelli, M., Xin, W., et al. (2020). Control of food approach and eating by a GABAergic projection from lateral hypothalamus to dorsal pons. Proc. Natl. Acad. Sci. U.S.A. doi:10.1073/pnas.1909340117.

Mathis, A., Mamidanna, P., Cury, K. M., Abe, T., Murthy, V. N., Mathis, M. W., et al. (2018). DeepLabCut: markerless pose estimation of user-defined body parts with deep learning. Nat. Neurosci. 21, 12811289. doi:10.1038/s41593-018-0209-y.

Mendelson, J. (1967). Lateral hypothalamic stimulation in satiated rats: the rewarding effects of self-induced drinking. Science 157, 1077-1079. doi:10.1126/science.157.3792.1077.

Mickelsen, L. E., Bolisetty, M., Chimileski, B. R., Fujita, A., Beltrami, E. J., Costanzo, J. T., et al. (2019). Single-cell transcriptomic analysis of the lateral hypothalamic area reveals molecularly distinct populations of inhibitory and excitatory neurons. Nat. Neurosci. 22, 642-656. doi:10.1038/s41593019-0349-8.

Mogenson, G. J., and Stevenson, J. A. (1967). Drinking induced by electrical stimulation of the lateral hypothalamus. Experimental neurology 17, 119-27. doi:10.1016/0014-4886(67)90139-2.

Nath, T., Mathis, A., Chen, A. C., Patel, A., Bethge, M., and Mathis, M. W. (2019). Using DeepLabCut for 3D markerless pose estimation across species and behaviors. Nat Protoc 14, 2152-2176. doi:10.1038/s41596-019-0176-0.

Navarro, M., Olney, J. J., Burnham, N. W., Mazzone, C. M., Lowery-Gionta, E. G., Pleil, K. E., et al. (2016). Lateral Hypothalamus GABAergic Neurons Modulate Consummatory Behaviors Regardless of the Caloric Content or Biological Relevance of the Consumed Stimuli. Neuropsychopharmacology: official publication of the American College of Neuropsychopharmacology 41, 1505-12. doi:10.1038/npp.2015.304.

Nieh, E. H., Matthews, G. A., Allsop, S. A., Presbrey, K. N., Leppla, C. A., Wichmann, R., et al. (2015). Decoding neural circuits that control compulsive sucrose seeking. Cell 160, 528-541. doi:10.1016/j.cell.2015.01.003.

Nieh, E. H., Vander Weele, C. M., Matthews, G. A., Presbrey, K. N., Wichmann, R., Leppla, C. A., et al. (2016). Inhibitory Input from the Lateral Hypothalamus to the Ventral Tegmental Area Disinhibits Dopamine Neurons and Promotes Behavioral Activation. Neuron 90, 1286-1298. doi:10.1016/j.neuron.2016.04.035.

Norgren, R. (1970). Gustatory responses in the hypothalamus. Brain Res. 21, 63-77. doi:10.1016/00068993(70)90021-1. 
976

977

978

979

980

981

982

983

984

985

986

987

988

989

990

991

992

993

994

995

996

997

998

999

1000

1001

1002

1003

1004

1005

1006

1007

1008

1009

1010

1011

1012

1013

O’Connor, E. C., Kremer, Y., Lefort, S., Harada, M., Pascoli, V., Rohner, C., et al. (2015). Accumbal D1R Neurons Projecting to Lateral Hypothalamus Authorize Feeding. Neuron 88, 553-564. doi:10.1016/j.neuron.2015.09.038.

Olds, J., and Milner, P. (1954). Positive reinforcement produced by electrical stimulation of septal area and other regions of rat brain. J Comp Physiol Psychol 47, 419-427. doi:10.1037/h0058775.

Ono, T., Nakamura, K., Nishijo, H., and Fukuda, M. (1986). Hypothalamic neuron involvement in integration of reward, aversion, and cue signals. J. Neurophysiol. 56, 63-79. doi:10.1152/jn.1986.56.1.63.

Phillips, A. G., and Mogenson, G. J. (1968). Effects of taste on self-stimulation and induced drinking. J Comp Physiol Psychol 66, 654-660. doi:10.1037/h0026523.

Poschel, B. P. H. (1968). Do biological reinforcers act via the self-stimulation areas of the brain? Physiology \& Behavior 3, 53-60. doi:10.1016/0031-9384(68)90031-0.

Qualls-Creekmore, E., Yu, S., Francois, M., Hoang, J., Huesing, C., Bruce-Keller, A., et al. (2017). GalaninExpressing GABA Neurons in the Lateral Hypothalamus Modulate Food Reward and Noncompulsive Locomotion. J. Neurosci. 37, 6053-6065. doi:10.1523/JNEUROSCI.0155-17.2017.

Schwartzbaum, J. S. (1988). Electrophysiology of taste, feeding and reward in lateral hypothalamus of rabbit. Physiol. Behav. 44, 507-526. doi:10.1016/0031-9384(88)90313-7.

Sclafani, A. (1991). "The Hedonics of Sugar and Starch.," in The hedonics of taste. (New York, NY.: Lawrence Erlbaum Associates, Inc), 59-87.

Sharpe, M. J., Marchant, N. J., Whitaker, L. R., Richie, C. T., Zhang, Y. J., Campbell, E. J., et al. (2017). Lateral Hypothalamic GABAergic Neurons Encode Reward Predictions that Are Relayed to the Ventral Tegmental Area to Regulate Learning. Current Biology 27, 2089-2100.e5. doi:10.1016/j.cub.2017.06.024.

Simerly, R. B. (2004). Anatomical Substrates of Hypothalamic Integration. In: The Rat Nervous System. Academic Press.

Spector, A. C., Klumpp, P. A., and Kaplan, J. M. (1998). Analytical issues in the evaluation of food deprivation and sucrose concentration effects on the microstructure of licking behavior in the rat. Behavioral neuroscience 112, 678-94.

Stuber, G. D., and Wise, R. A. (2016). Lateral hypothalamic circuits for feeding and reward. Nature neuroscience 19, 198-205. doi:10.1038/nn.4220.

Teitelbaum, P., and Epstein, A. N. (1962). The lateral hypothalamic syndrome: recovery of feeding and drinking after lateral hypothalamic lesions. Psychological review 69, 74-90.

Thoeni, S., Loureiro, M., O'Connor, E. C., and Lüscher, C. (2020). Depression of Accumbal to Lateral Hypothalamic Synapses Gates Overeating. Neuron. doi:10.1016/j.neuron.2020.03.029.

Ting, J. T., and Feng, G. (2013). Development of transgenic animals for optogenetic manipulation of mammalian nervous system function: progress and prospects for behavioral neuroscience. Behav. Brain Res. 255, 3-18. doi:10.1016/j.bbr.2013.02.037.

Urstadt, K. R., and Berridge, K. C. (2020). Optogenetic mapping of feeding and self-stimulation within the lateral hypothalamus of the rat. PLOS ONE 15, e0224301. doi:10.1371/journal.pone.0224301. 
1014

1015

1016

1017

1018

1019

1020

1021

1022

1023

1024

1025

1026

1027

1028

1029

1030

1031

1032

1033

1034

1035

1036

1037
Valenstein, E. S., and Cox, V. C. (1970). Influence of hunger, thirst, and previous experience in the test chamber on stimulus-bound eating and drinking. J Comp Physiol Psychol 70, 189-199. doi: $10.1037 / \mathrm{h} 0028708$.

Valenstein, E. S., Cox, V. C., and Kakolewski, J. W. (1968). Modification of Motivated Behavior Elicited by Electrical Stimulation of the Hypothalamus. Science 159, 1119-1121. doi:10.1126/science.159.3819.1119.

Valenstein, E. S., and Phillips, A. G. (1970). Stimulus-bound eating and deprivation from prior contact with food pellets. Physiology \& Behavior 5, 279-282. doi:10.1016/0031-9384(70)90099-5.

Villavicencio, M., Moreno, M. G., Simon, S. A., and Gutierrez, R. (2018). Encoding of Sucrose's Palatability in the Nucleus Accumbens Shell and Its Modulation by Exteroceptive Auditory Cues. Front Neurosci 12, 265. doi:10.3389/fnins.2018.00265.

Yamamoto, T., Matsuo, R., Kiyomitsu, Y., and Kitamura, R. (1989). Response properties of lateral hypothalamic neurons during ingestive behavior with special reference to licking of various taste solutions. Brain Res. 481, 286-297. doi:10.1016/0006-8993(89)90805-6.

Zeng, H., and Madisen, L. (2012). Mouse transgenic approaches in optogenetics. Prog. Brain Res. 196, 193213. doi:10.1016/B978-0-444-59426-6.00010-0.

Zhang, X., and van den Pol, A. N. (2017). Rapid binge-like eating and body weight gain driven by zona incerta GABA neuron activation. Science 356, 853-859. doi:10.1126/science.aam7100.

Zhao, S., Ting, J. T., Atallah, H. E., Qiu, L., Tan, J., Gloss, B., et al. (2011). Cell type-specific channelrhodopsin-2 transgenic mice for optogenetic dissection of neural circuitry function. Nature methods 8, 745-52.

Zocchi, D., Wennemuth, G., and Oka, Y. (2017). The cellular mechanism for water detection in the mammalian taste system. Nat. Neurosci. 20, 927-933. doi:10.1038/nn.4575. 

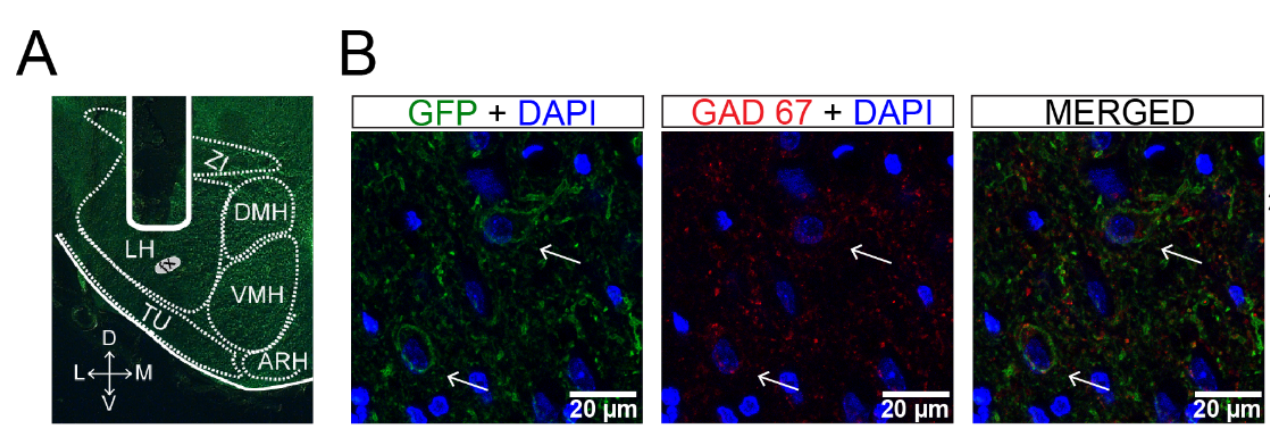

C fcanner of

D

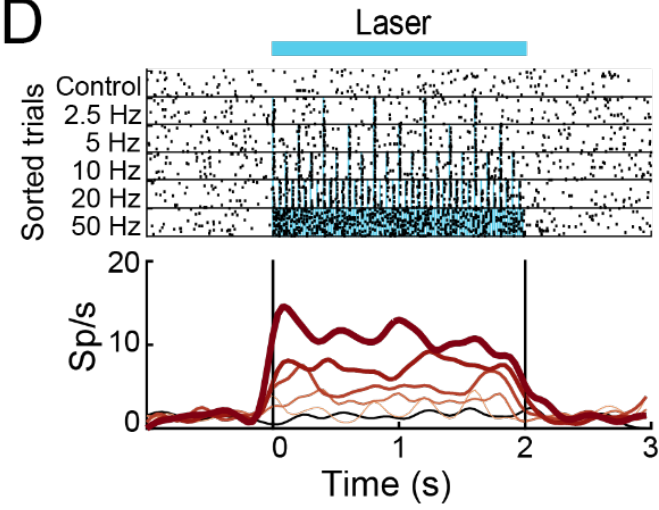

$\mathrm{E}$

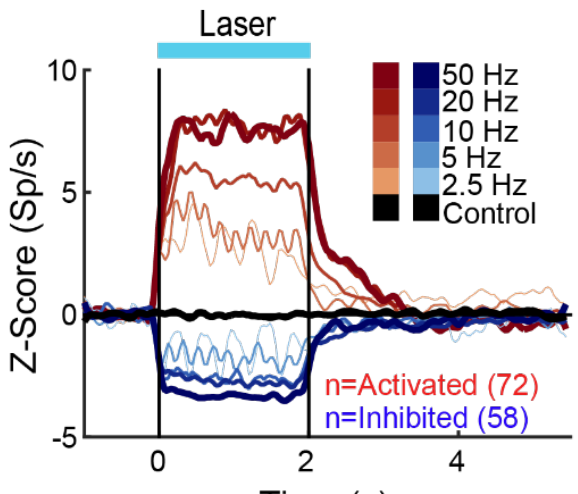

1038

1039

1040

1041

1042

1043

1044

1045

1046

1047

1048

1049

1050

1051

1052

1053

Time (s)

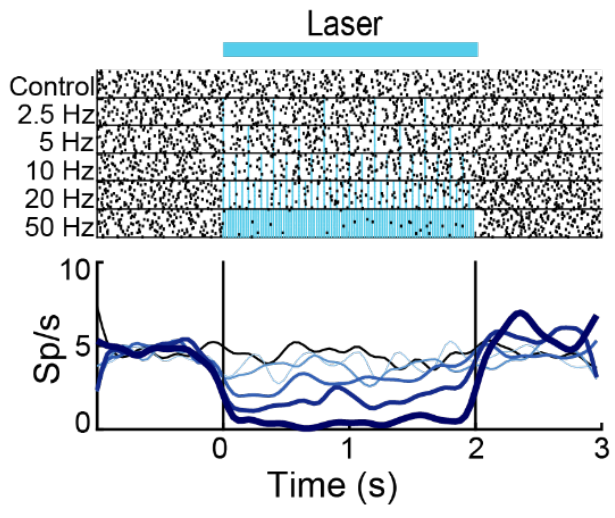

$\mathrm{F}$

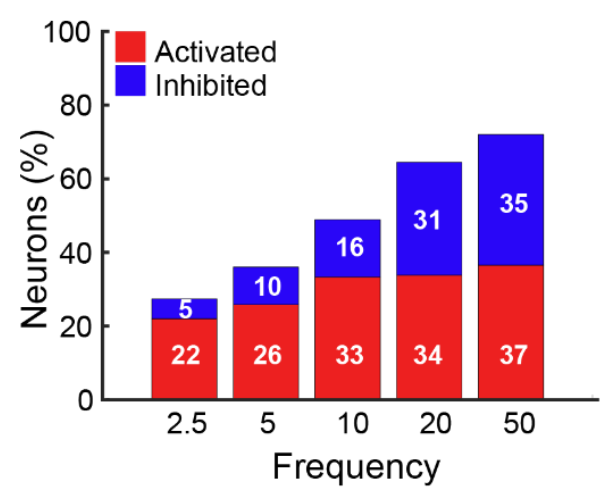

Figure 1. The $50 \mathrm{~Hz}$ stimulation evoked the largest LHA neuronal responses. (A) Representative location of an optrode in LHA of VGAT-ChR2 mice. (B) Confocal images from LHA immunostained for GFP (green), GAD67 (red), and DAPI (blue). Arrows indicate the colocalization of ChR2-EYFP and GAD67 (see merged). (C) Schematic of optrode recordings in LHA during control trials (with nolaser) and opto-stimulation at 2.5, 5, 10,20, and $50 \mathrm{~Hz}$ delivered randomly. In each trial, the laser had a cycle of $2 \mathrm{~s}$ on and $4 \mathrm{~s}$ off. (D) Representative raster plots of two neurons recorded in LHA. The first one was activated (left panel), and the second inhibited (right panel) during opto-stimulation. Spiking responses were aligned (Time $=0 \mathrm{~s}$ ) to the first laser pulse. Black ticks depict action potentials and blue marks laser pulses. Below are the corresponding PSTHs (firing rates, $\mathrm{Sp} / \mathrm{s}$ ). Red and blue ascending gradients indicate the frequency of stimulation (see E for the color bar). Black trace represents activity during control trials without laser. Vertical black lines indicate laser onset (Time $=$ $0 \mathrm{~s}$ ) and offset (Time $=2 \mathrm{~s}$ ), respectively. (E) Normalized Z-score population responses of LHA neurons recorded from $\mathrm{n}=5$ VGAT-ChR2 mice. Red and blue colors depict activated or inhibited responses, respectively, relative to baseline ( -1 to $0 \mathrm{~s}$ ), thickness, and gradient colors indicate higher 
bioRxiv preprint doi: https://doi.org/10.1101/2020.06 30.180646. this version posted December 27,2020 . The copyright holder for this preprint (which was not certified by peer review) is the author/funder, who has granted bioRxiv a license to display the preprint in perpetuity. It is made available under aCC-BY-NC-ND 4.0 interpatiend Bensergic neurons encode palatability

1054 stimulation frequencies. Black trace illustrates neural activity during control trials for all recorded 1055 neurons $(\mathrm{n}=186)$. (F) Percentage of neurons recruited as a function of stimulation frequency.

1056 
bioRxiv preprint doi: https://doi.org/10.1101/2020.06.30.180646; this version posted December 27, 2020. The copyright holder for this preprint (which was not certified by peer review) is the author/funder, who has granted bioRxiv a license to display the preprint in perpetuity. It is made

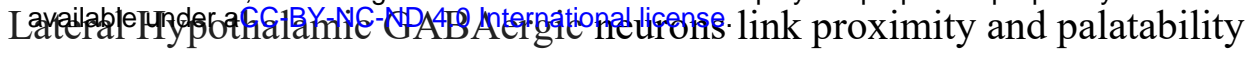

A

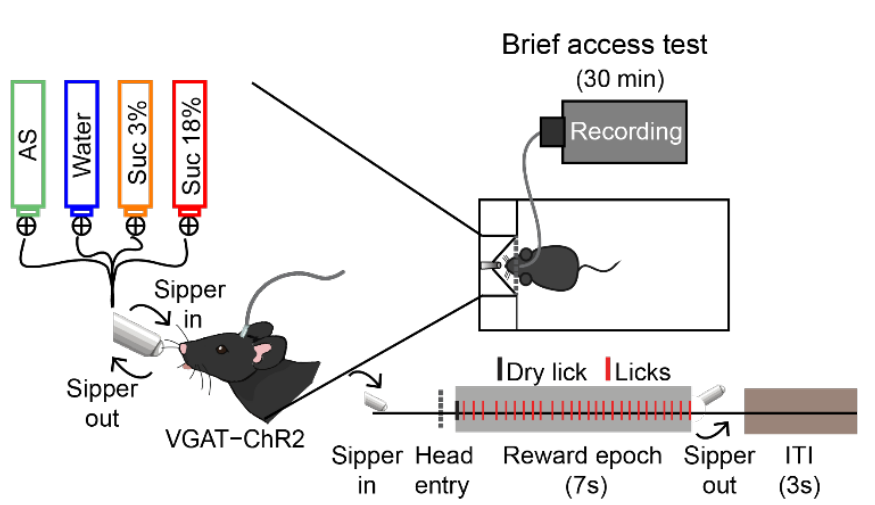

B

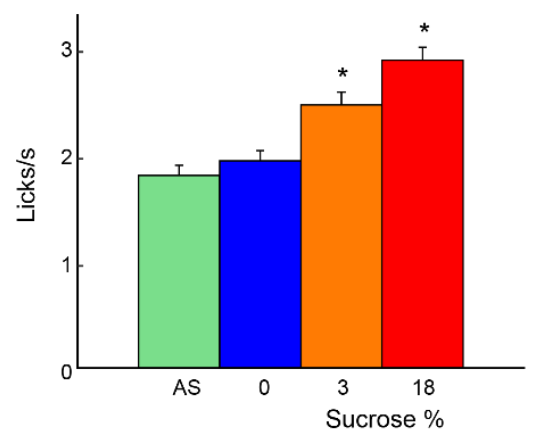

C
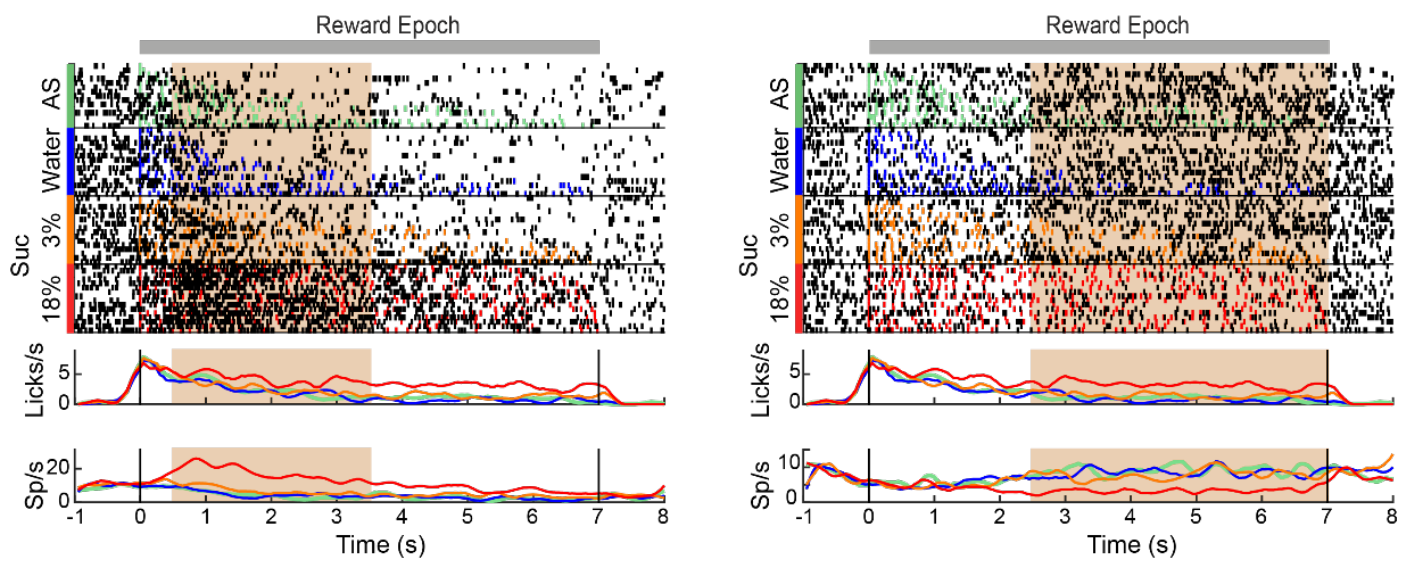

D

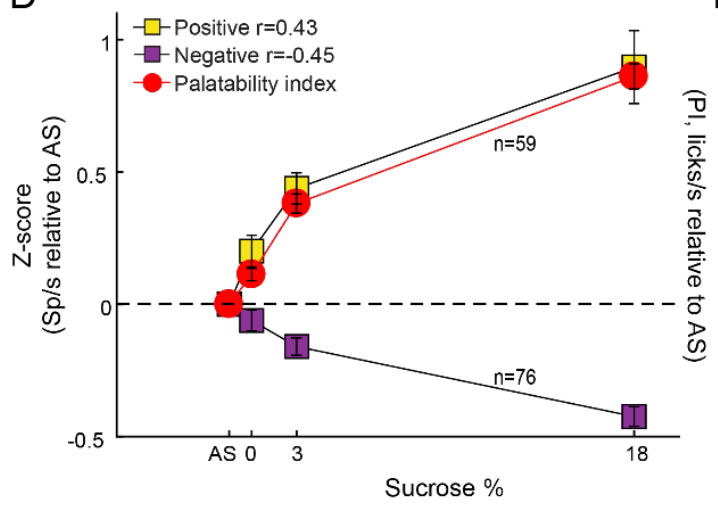

E

F
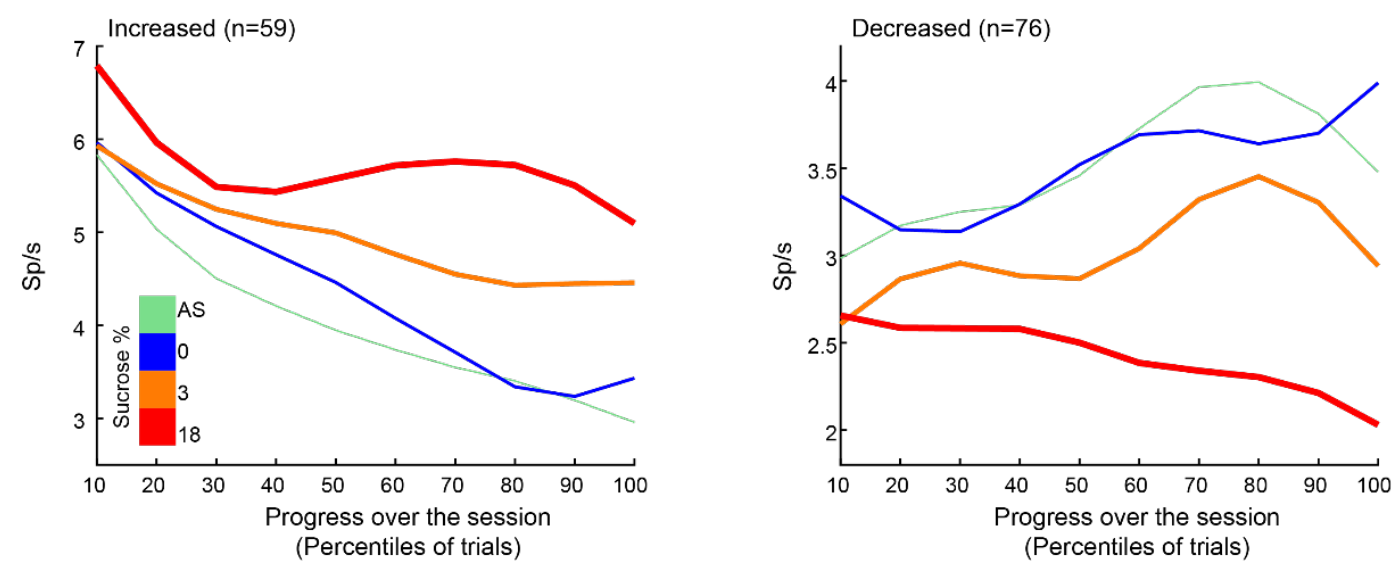
1058 Figure 2. LHA neurons process palatability-related information. (A) Brief access taste setup depicting 1059 a behavioral box equipped with a computer-controlled retractile sipper that for each lick independently 1060 delivers a drop of artificial saliva (AS), water, sucrose 3\%, or 18\% concentrations. After a head entry 1061 (dashed line), the first dry lick (black line) enables the $7 \mathrm{~s}$ reward epoch. During this period, in each 1062 lick (red), a drop of the four tastants was randomly delivered. After the reward epoch, the sipper was 1063 retracted for an Intertrial Interval (ITI) of $3 \mathrm{~s}$ and then re-extended to start a new trial. (B) Average lick 1064 rate during the entire reward epoch, reflecting greater palatability as a function of sucrose 1065 concentration. * Indicates significant difference $(\mathrm{p}<0.05)$ among stimuli. Using one-way ANOVA 1066 followed by the Holm Sidak test. (C) Representative raster plot of two LHA neurons recorded while 1067 mice performed a brief access test. Spikes are plotted as black ticks, whereas licks for each tastant are 1068 color-coded (green, AS; blue, water; orange, sucrose 3\%; red, sucrose 18\%). Below are corresponding 1069 peristimulus time histograms (PSTHs) of lick rate (Licks/s) and firing rate (Spikes/s; Sp/s) as a function 1070 of trial type. Neuronal responses were aligned (Time $=0 \mathrm{~s}$ ) to the first rewarded lick. The brown 1071 rectangles depict the "best" window with the maximum Pearson correlation coefficient between firing 1072 rates and sucrose's palatability index (PI). The left and right raster plots displayed two neurons with a 1073 positive and negative correlation, respectively. (D) Z-score normalized activity (relative to AS trials) 1074 for LHA neurons with either positive or negative correlation against PI (red circles). (E) Population 1075 PSTHs of the lick rates given in the Reward epoch as a function of trial type, divided into blocks of $107610^{\text {th }}$ percentiles of trials each. (F) Population PSTHs of the firing rate during the Reward epoch of the 1077 palatability-related neurons across the session for each trial type. Left panel depicts neurons that fired 1078 more to higher sucrose concentrations (positive sucrose's palatability correlation; (D) yellow), 1079 whereas, right panel illustrates neurons with decreasing firing rates as the sucrose concentration 1080 increased (i.e., negative correlation with sucrose's palatability; (D) purple). 

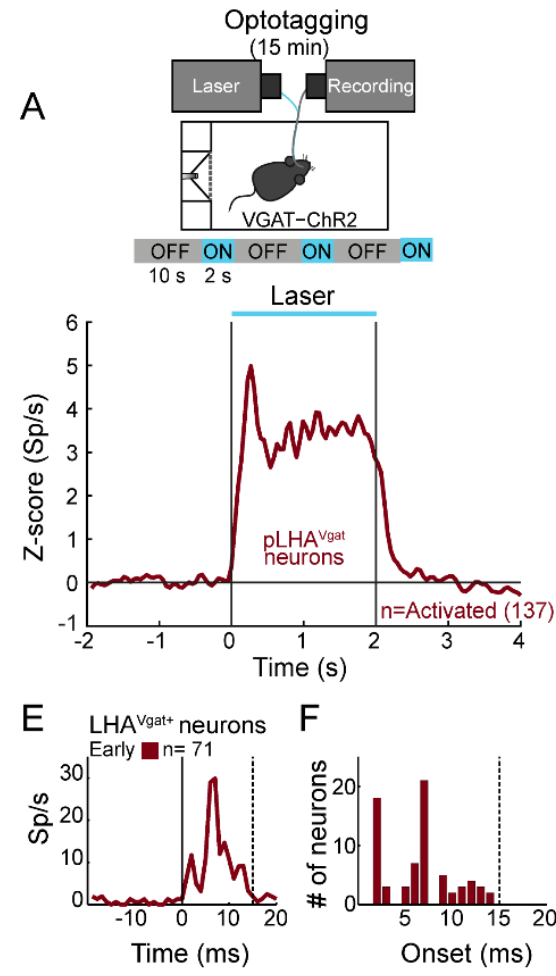

J

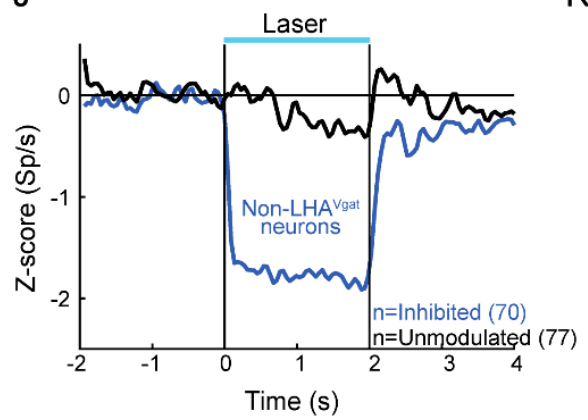

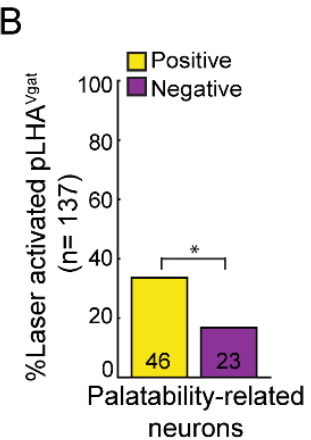

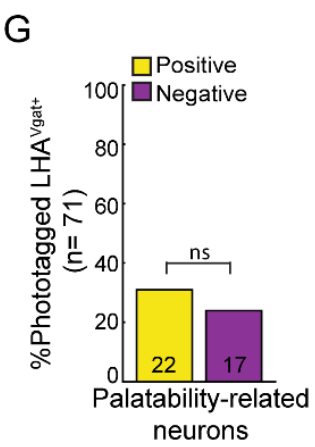

$\mathrm{K}$

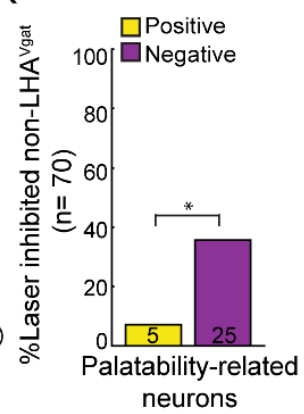

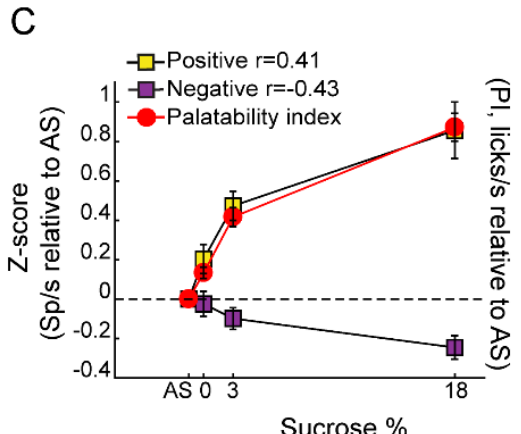

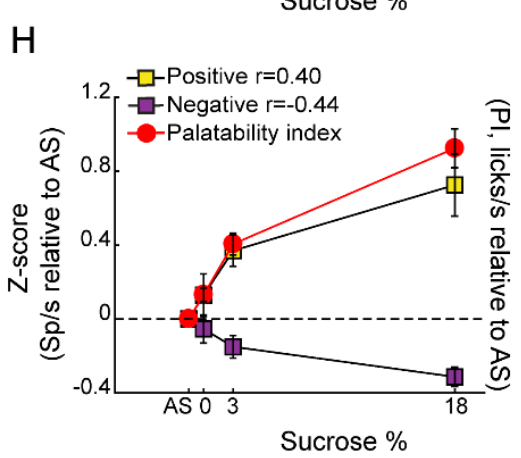

$\mathrm{L}$

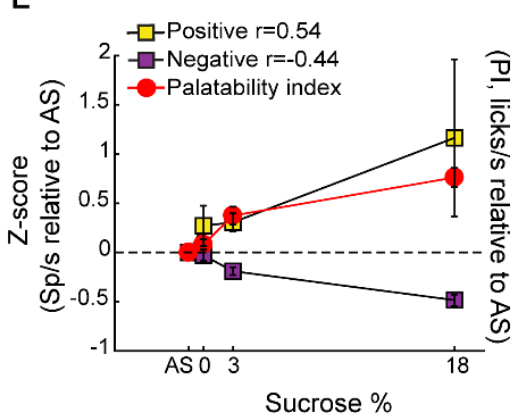

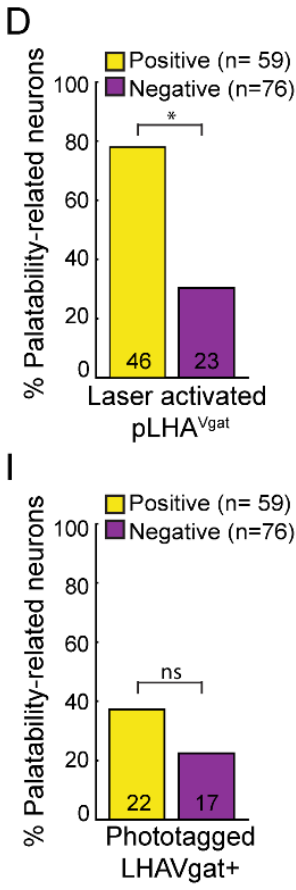

M

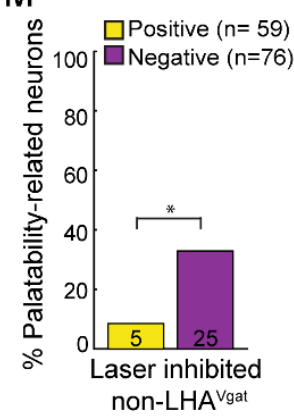

1082 Figure 3. A subpopulation of $\mathrm{LHA}^{\text {Vgat+ }}$ neurons encodes sucrose's palatability. (A) To confirm the LHA $^{\text {Vgat }}{ }^{+}$neurons' identity after the brief access test, we performed laser opto-stimulation in a cycle 2 $\mathrm{s}$ on (at $50 \mathrm{~Hz}$ ) and $10 \mathrm{~s}$ off over $15 \mathrm{~min}$ session. In this phase, mice had no access to the sipper (above panel). Below, the normalized Z-score population activity of all 284 LHA neurons recorded from 3 VGAT-ChR2 mice. Responses were aligned to the laser onset (Time $=0 \mathrm{~s})$, relative to baseline $(-1$ to $0 \mathrm{~s})$ activity. The red line depicts the responses of activated putative $\mathrm{pLHA}^{\text {Vgat }}$ neurons. (B) Percentage of laser-activated $\mathrm{pLHA}^{\mathrm{Vgat+}}$ neurons whose activity (in the brief access test) also positively or negatively correlates with sucrose's PI. * $p<0.01$, chi-square. (C) Z-score activity (relative to AS) for all activated $\mathrm{pLHA}^{\mathrm{Vgat}}$ neurons and its correlation with the sucrose's PI. (D) Percentage of Positive or Negative laser-activated palatability-related neurons. ${ }^{*} p<0.0001$, chi-square. (E) PSTH of identified (opto-tagged) $\mathrm{LHA}^{\mathrm{Vgat}+}$ neurons exhibiting early evoked responses (i.e., ChR2 expressing cells). (F) Histogram of opto-tagged $\mathrm{LHA}^{\mathrm{Vgat}+}$ neurons with evoked early responses (latencies $<15 \mathrm{~ms}$ ) from laser onset. (G-I) same conventions as in B-D, but for opto-tagged $\mathrm{LHA}^{\mathrm{Vgat}^{+}}$neurons encoding sucrose's palatability. (J-M) Non-LHA ${ }^{\text {Vgat }}$ neurons that negatively encode sucrose's palatability. $\boldsymbol{J}$, Blue and black traces correspond to Inhibited (Non-LHA ${ }^{\mathrm{Vgat}+}$ neurons) and Unmodulated responses, respectively. Same conventions as in $(A)$. (K-M) same conventions as in B-D, ${ }^{*} p<0.001$, chi-square. 
A

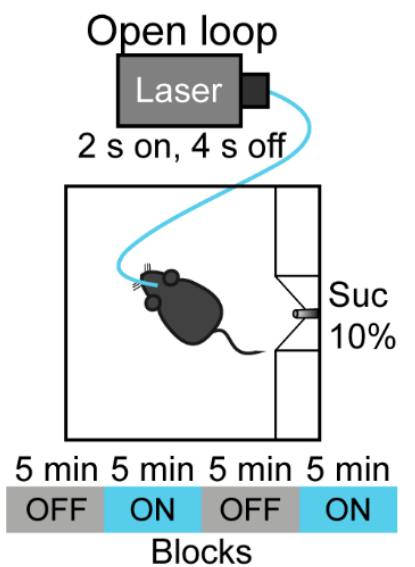

C

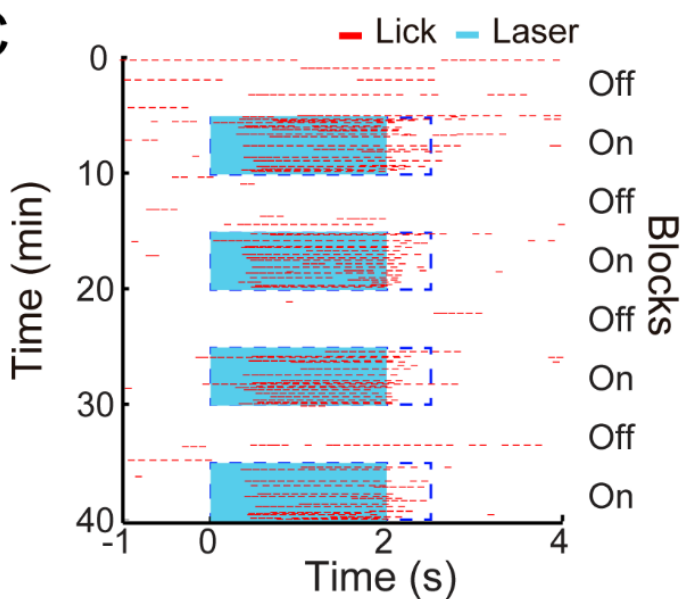

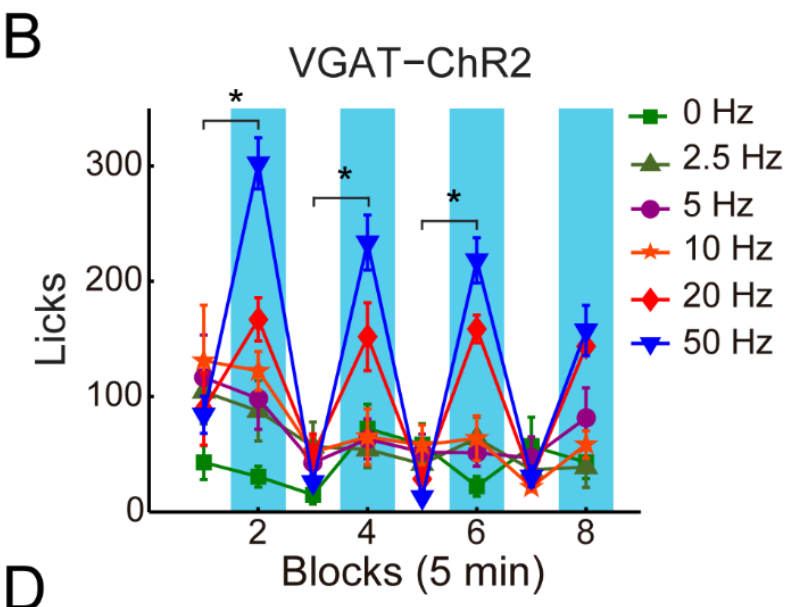

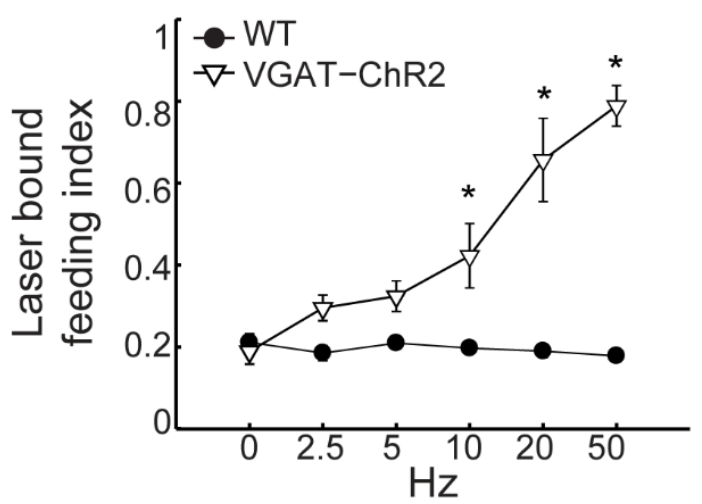

1100 Figure 4. In sated VGAT-ChR2 mice, $50 \mathrm{~Hz}$ stimulation of LHA $^{\text {Vgat }}{ }^{+}$neurons induced the greatest 1101 intake of sucrose. $\boldsymbol{A}$, Behavioral setup for open-loop stimulation, where the laser was turned on 1102 regardless of the mice's behavior. The box was equipped with a central sipper, where mice had free access to $10 \%$ sucrose. Open-loop stimulation comprised $5 \mathrm{~min}$ block without laser followed by optostimulation for $5 \mathrm{~min}$, in a cycle $2 \mathrm{~s}$ on $-4 \mathrm{~s}$ off, during a $40 \mathrm{~min}$ session. VGAT-ChR2 $(\mathrm{n}=3)$ and WT $(n=5)$ mice received stimulation of only one of the following frequencies in ascending order per session $0,2.5,5,10,20$, and $50 \mathrm{~Hz}$. B, Opto-stimulation of LHA ${ }^{\text {Vgat+ }}$ neurons induced sucrose $(10 \%)$ intake in a frequency-dependent manner that peaked at $50 \mathrm{~Hz} .{ }^{*}$ Denotes statistically significant difference $(p<0.001)$ between non-laser and laser stimulated blocks at $50 \mathrm{~Hz}$. Besides mice were sated, the lower intake at $0 \mathrm{~Hz}$ could be due to the lack of experience drinking in the novel box. $\boldsymbol{C}$, Representative raster plot of open-loop stimulation from a VGAT-ChR2 mouse at $50 \mathrm{~Hz}$. Lick responses (red ticks) were aligned to laser onset (blue rectangles, opto-stimulation period). The laser bound index was the number of licks within $2.5 \mathrm{~s}$ from laser onset (dashed blue rectangles) divided by total licks. $\boldsymbol{D}$, The laser bound feeding index of VGAT-ChR2 increased as a function of the laser frequency. * Indicates significant difference from WT mice $(p<0.01)$. Using a Two-way ANOVA 

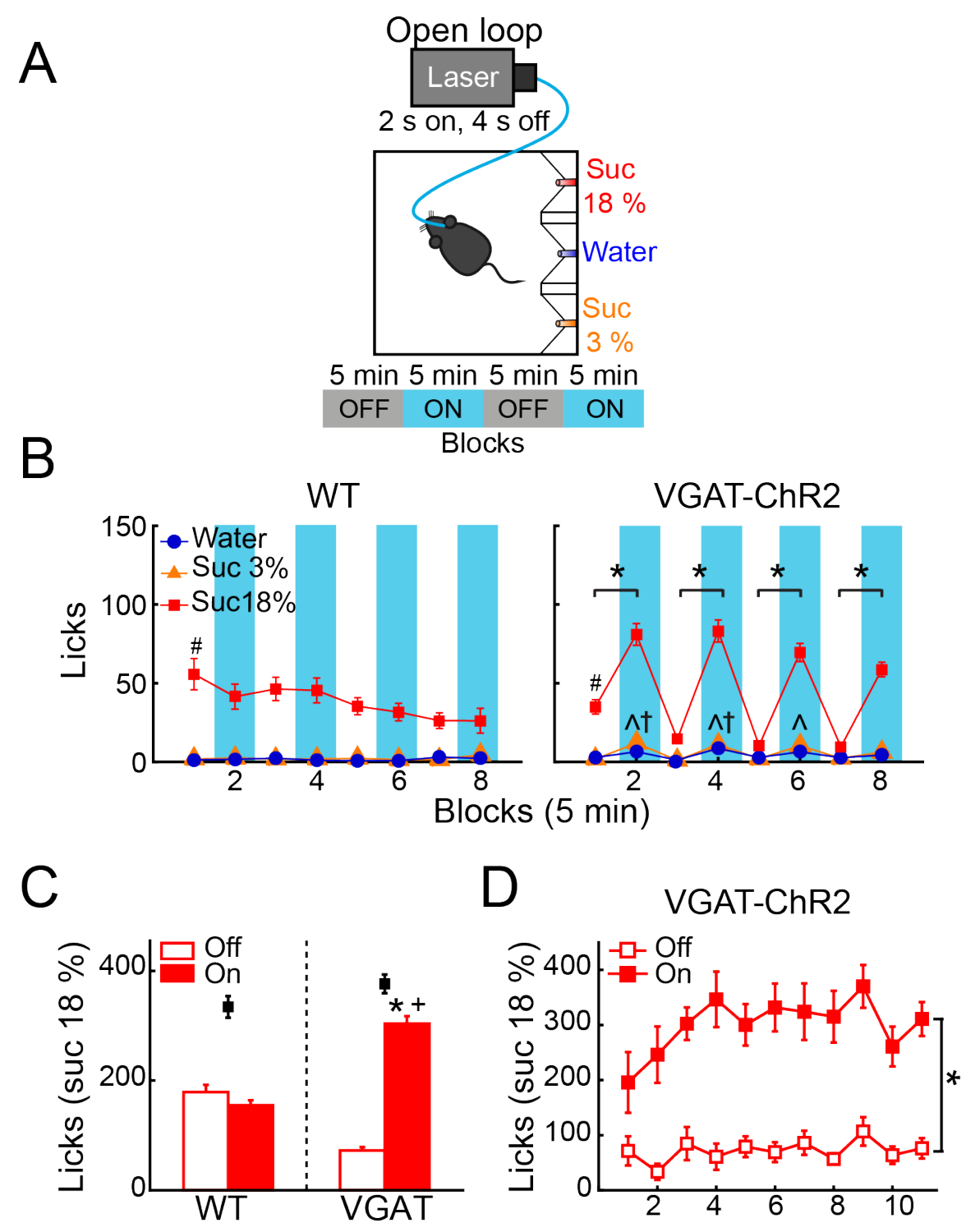

Days

1117 Figure 5. Open loop stimulation of LHA ${ }^{\mathrm{Vgat}+}$ neurons biases consumption towards the most palatable tastant and reinforce intake during opto-stimulation. (A) Behavioral protocol. Sated mice $(\mathrm{n}=6 \mathrm{WT}$ and $n=12$ VGAT-ChR2) were located in an operant box equipped with three ports. The left port contained sucrose $18 \%$, the central port water, and the right port sucrose $3 \%$, counterbalanced across individuals. For details of open loop, see Figure 4A. (B) Left and right panel, number of licks for each gustatory stimulus for WT and VGAT-ChR2 mice. Note that the VGAT-ChR2 mice increased consumption of the most palatable stimulus available (sucrose 18\%) mainly in opto-stimulated blocks (blue rectangles), while it decreased intake in blocks without laser. (C) Mean licks for sucrose 18\%, during blocks with laser on and off from WT and VGAT-ChR2 mice. Black squares show the total licks given for each group. (D) Licks for sucrose 18\% during blocks with or without laser across days. * Indicates a significant difference $(p<0.0001)$ between laser and no-laser blocks. $\# p<0.0001$ statistically significant difference between sucrose $18 \%$ and the other stimuli. ${ }^{\wedge} p<0.01$ between WT and VGAT-ChR2 for sucrose 3\%; †p<0.01 higher intake of water of VGAT-ChR2 compared with WT; $+\mathrm{p}<0.0001$ higher consumption of VGAT-ChR2 compared with WT for sucrose $18 \%$ during blocks with laser. Using a two-way ANOVA and RM ANOVA followed by the Holm Sidak test. 
A

Suc $3 \%$

B

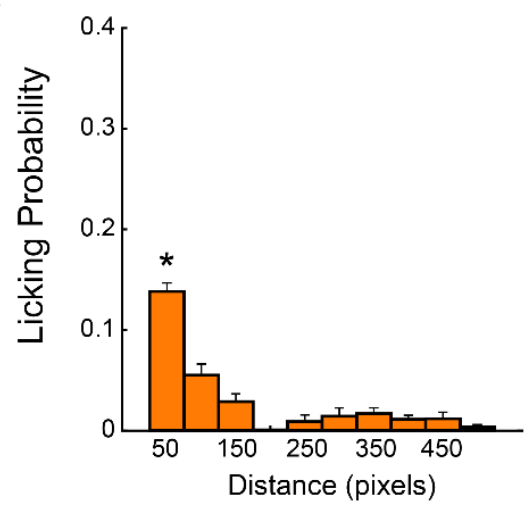

C
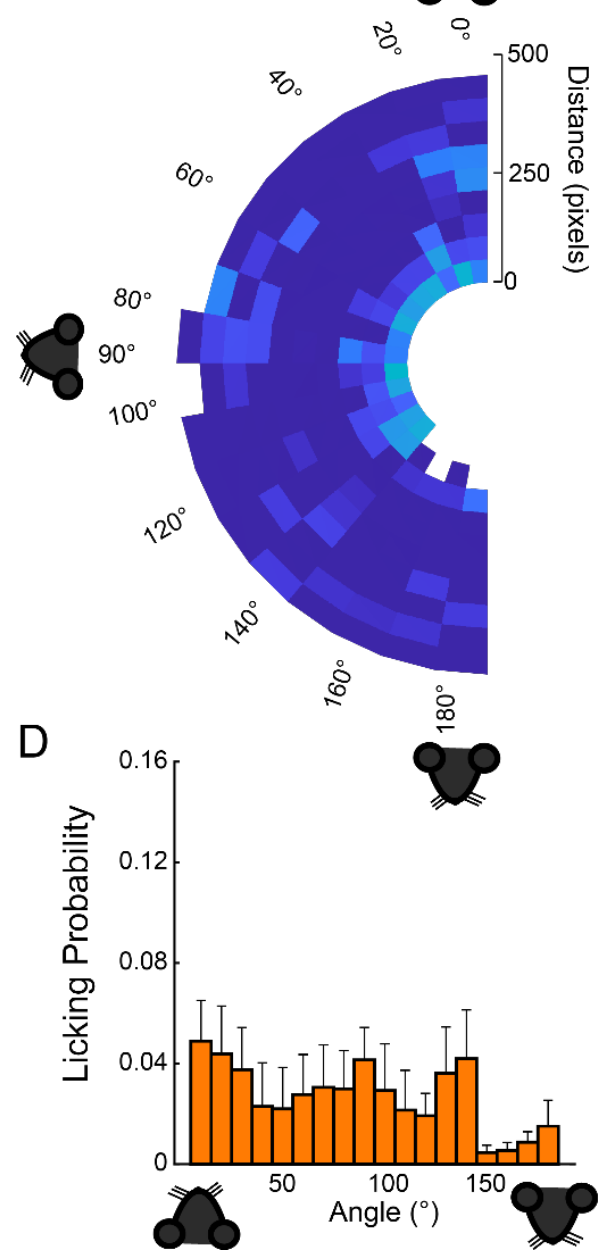

Water

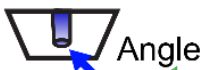

Distance
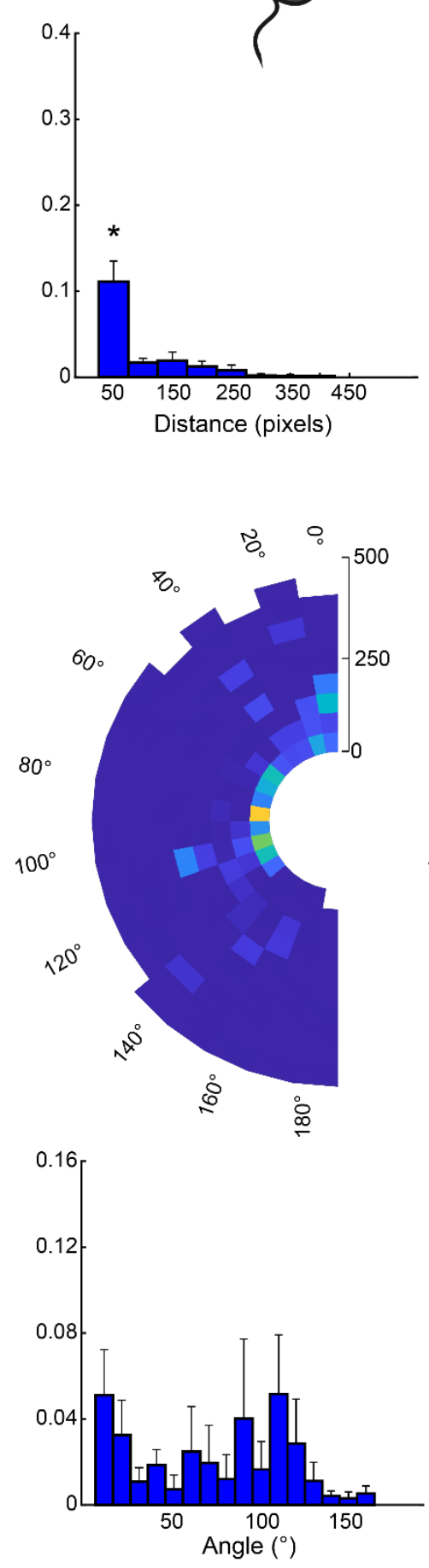

Suc $18 \%$
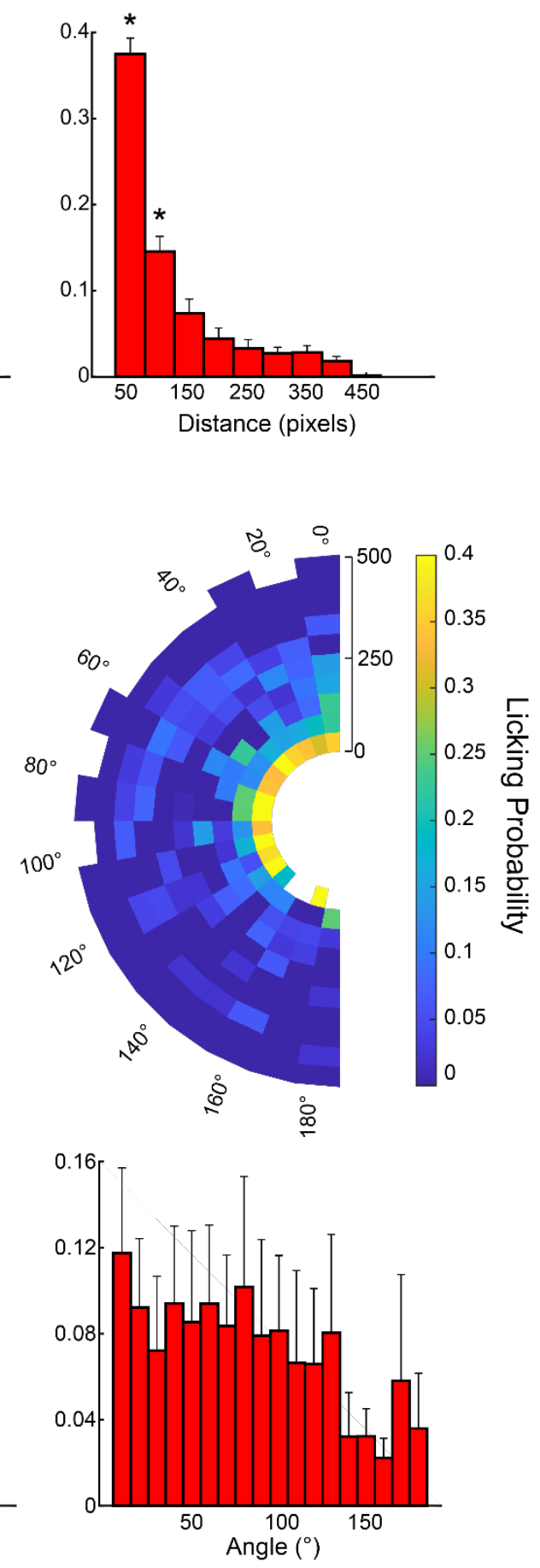

1133 Figure 6. Licking probability increased as a function of distance. (A) Schematics for video tracking 1134 (see Materials and Methods). We calculated the distance and the head angle between the mouse's nose 
1135 and each of the three sippers containing sucrose $3 \%$, water, or sucrose $18 \%$ for each laser onset. (B) It depicts the probability that laser onset evoked licking as a function of the mouse's distance relative to each sipper. (C) Polar plots depict the licking probability after laser onset, given the distance and angle of the mouse relative to a licking sipper. At the onset of each laser stimulation, we computed the head angle and distance relative to each sipper, and, in the following $6 \mathrm{~s}$ window, we tally if the mouse licked to any sipper. We normalized by dividing the number of times licking was elicited by the number of first lasers occurring on those coordinates. The drawing of the mice's head illustrates that if a mouse was facing towards the sipper, the angle is $0^{\circ}$, in a perpendicular position $\left(90^{\circ}\right)$, or $180^{\circ}$ if it was looking in the opposite direction. (D) The probability of licking after laser onset versus the angle between head direction and the sipper. * Indicates significantly different $(p<0.001)$ from all other distances. Oneway ANOVA followed by the Holm Sidak test.

A

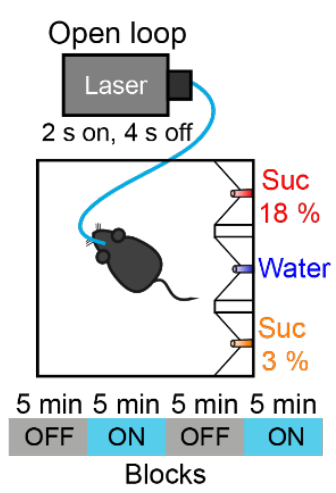

C

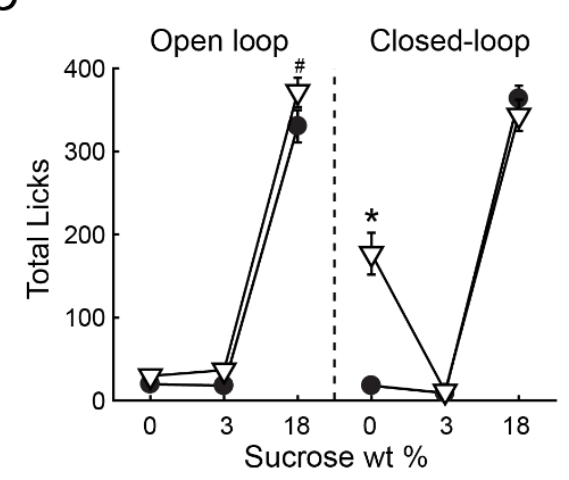

$\mathrm{F}$

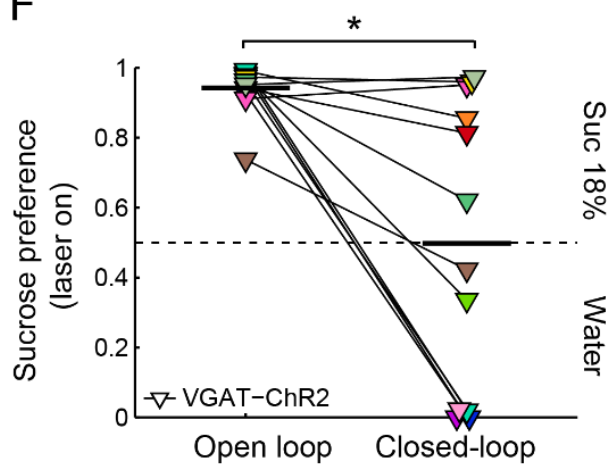

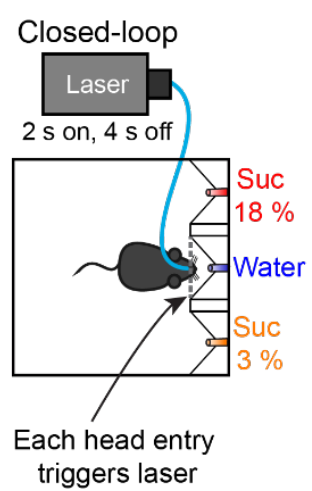

D

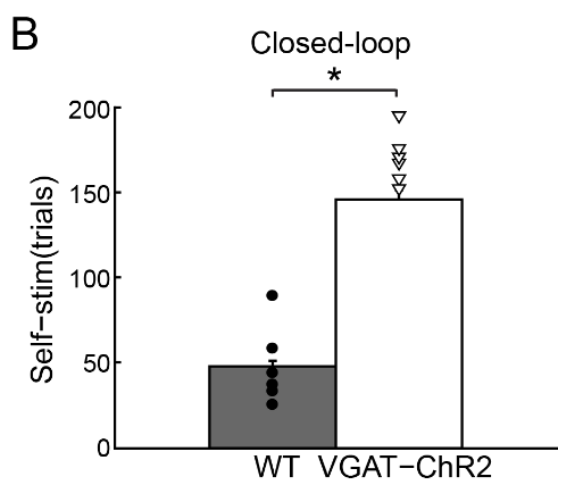

E

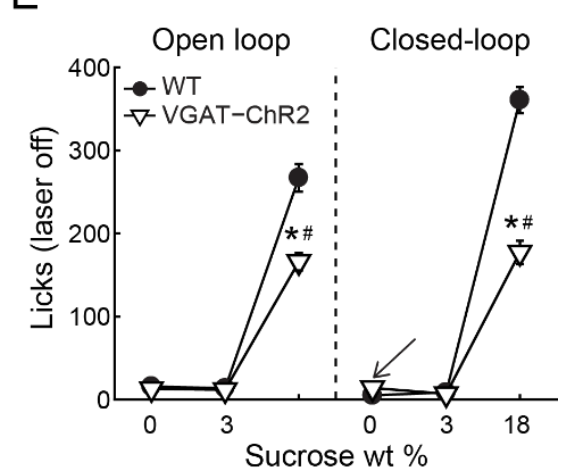

G
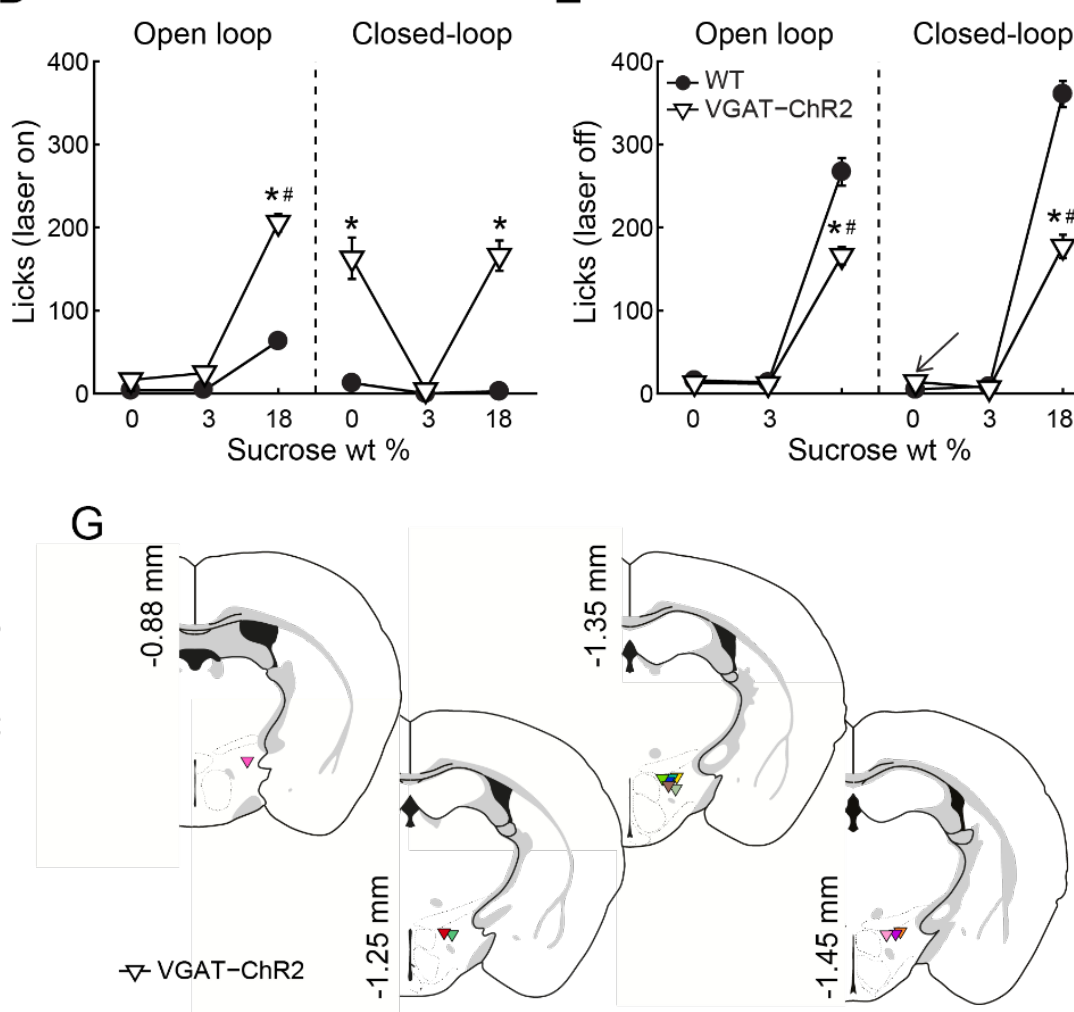

1148 Figure 7. If stimulation occurred near the less palatable stimulus, LHA ${ }^{\text {Vgat+ }}$ neurons induced water 1149 intake despite sucrose. (A) For the open loop stimulation, the laser was activated (2s on, 4s off) 
1150 regardless of behavior and mice position. Same mice and conventions as in Figure $5 A$. In the closed1151 loop configuration, the laser was triggered by a head entry in the central port. (B) The number of laser 1152 activations during the closed-loop protocol. The VGAT-ChR2 mice self-stimulate more than the WT 1153 mice. Each dot and triangle represent a single mouse. Unpaired t-test. (C) The total number of licks 1154 given for each stimulus during the entire session. In the open loop both mice groups licked more 1155 sucrose $18 \%$ than water or sucrose 3\%. However, in the closed-loop configuration, the VGAT-ChR2 1156 consumed more water (0\% sucrose) than the WT mice. (D) The number of licks evoked during $2.5 \mathrm{~s}$ 1157 after laser onset for each gustatory stimulus. (E) The number of licks when laser was turned off for 1158 each gustatory stimulus. (F) The water-sucrose $18 \%$ preference index from VGAT-ChR2 mice during 1159 photostimulation was defined as the number of $18 \%$ sucrose licks divided by total licks for sucrose $116018 \%$ + water. Thus, values higher than 0.5 indicate sucrose $18 \%$, and values lower than 0.5 prefer 1161 water. Some VGAT-ChR2 mice preferred water over sucrose. The horizontal black lines indicate mean 1162 preference in both stimulation protocols. Paired t-test. (G) Fiber optic location in the LHA of VGAT1163 ChR2 mice. ${ }^{*} p<0.0001$ indicates a significant difference from WT mice and stimulation protocols. \# $1164 p<0.0001$ between sucrose $18 \%$ and the other stimuli. Two-way ANOVA followed by the Holm 1165 Sidak test.

1166

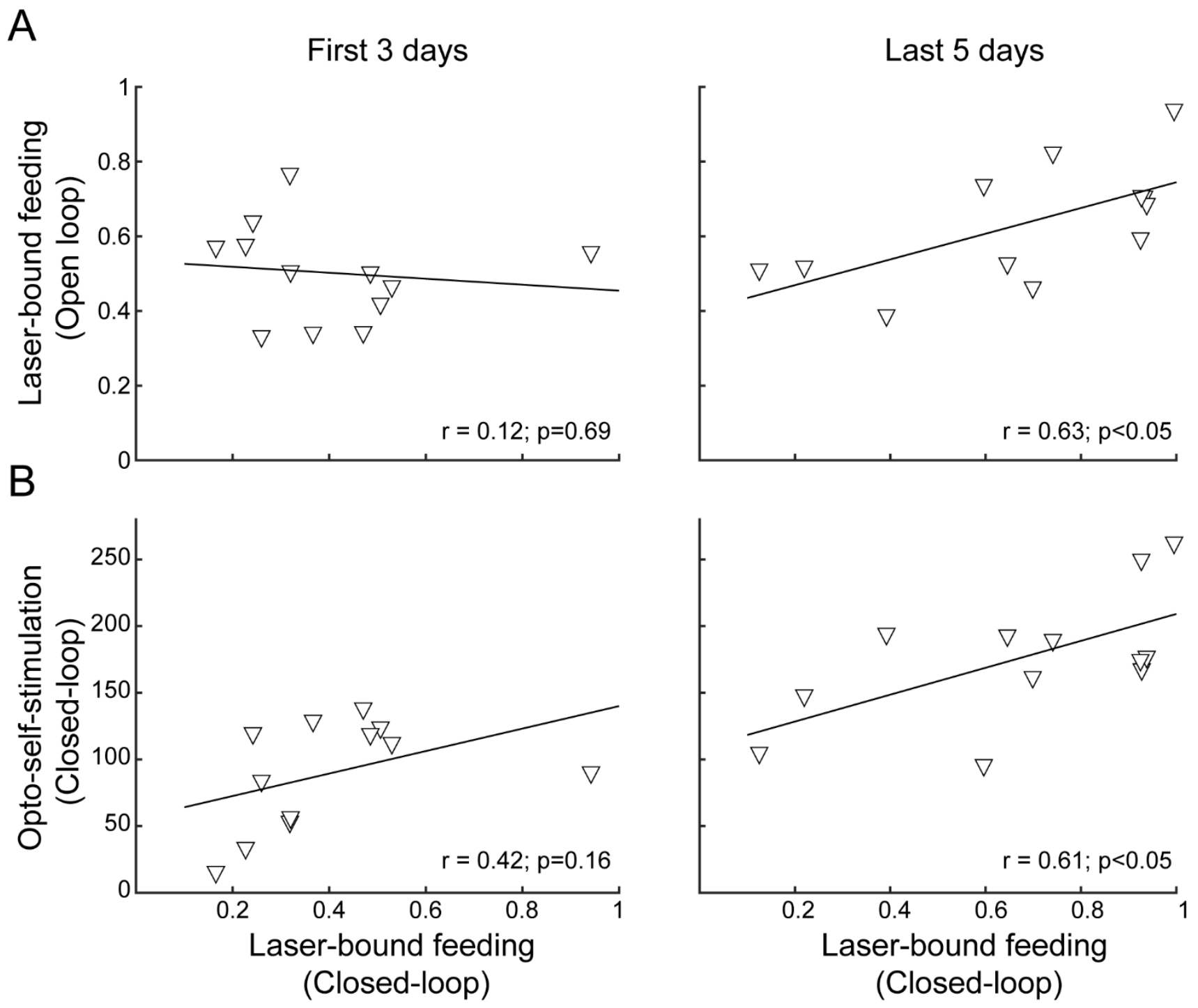


1168 Figure 8. After repeated activation of LHA ${ }^{\mathrm{Vgat}+}$ neurons, laser-bound feeding (in open vs. closed-loop) strengthened and exhibited a positive correlation. (A) Scatter plots showing the relationship between laser-bound feeding (licks given during the $2.5 \mathrm{~s}$ window from laser onset relative to total licks in the session) in both open loop and closed-loop tasks during the first 3 days (left panel) and the last 5 days of opto-stimulation of open loop vs. opto-self-stimulation of the closed-loop protocols (right panel). (B) Likewise, after repeated activation, both opto-self-stimulations and laser-bound feeding (licking) showed a significant correlation. Scatter depicting the average number of opto-self-stimulations vs. the laser-bound feeding observed in the closed-loop protocol.

A

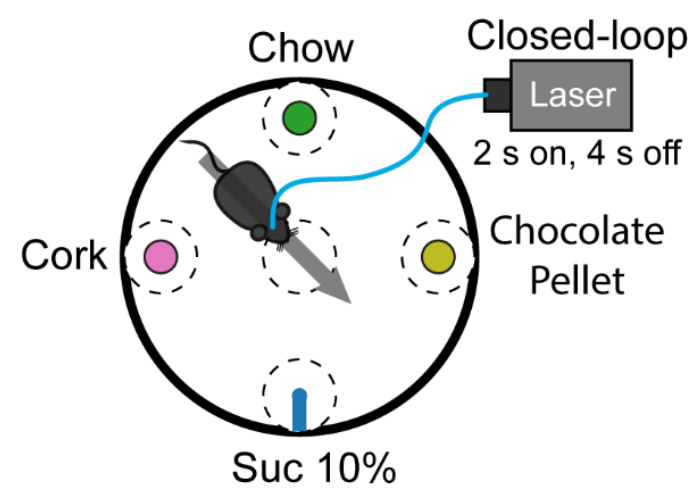

\section{B}

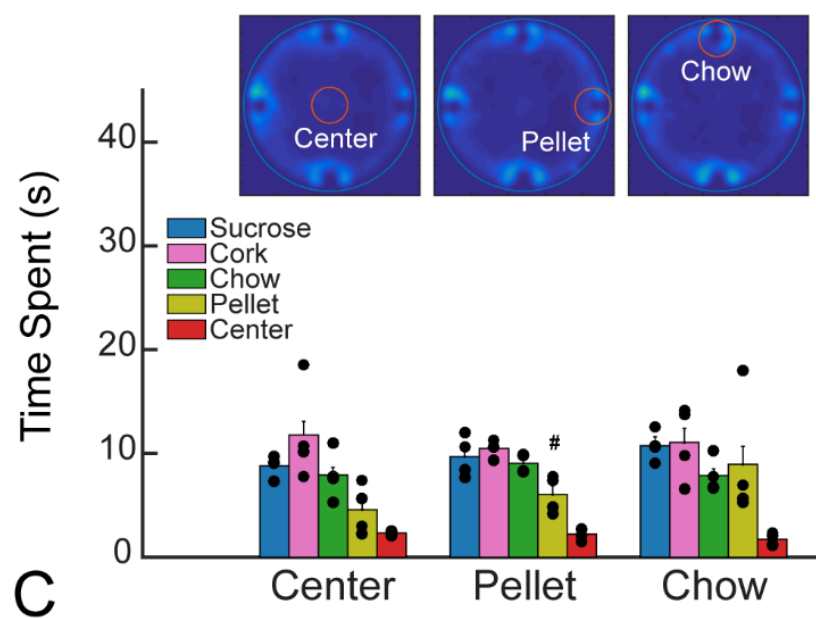

C

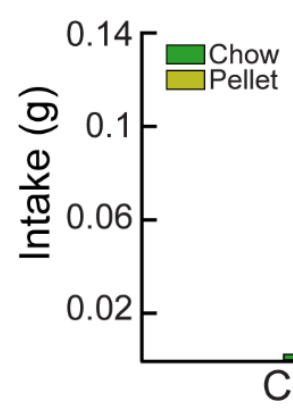

WT $(n=4)$

Pellet

Chow

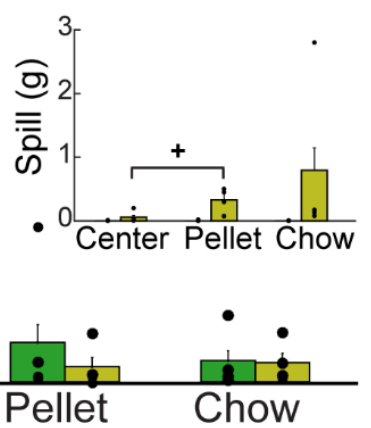

1177

1178

1179
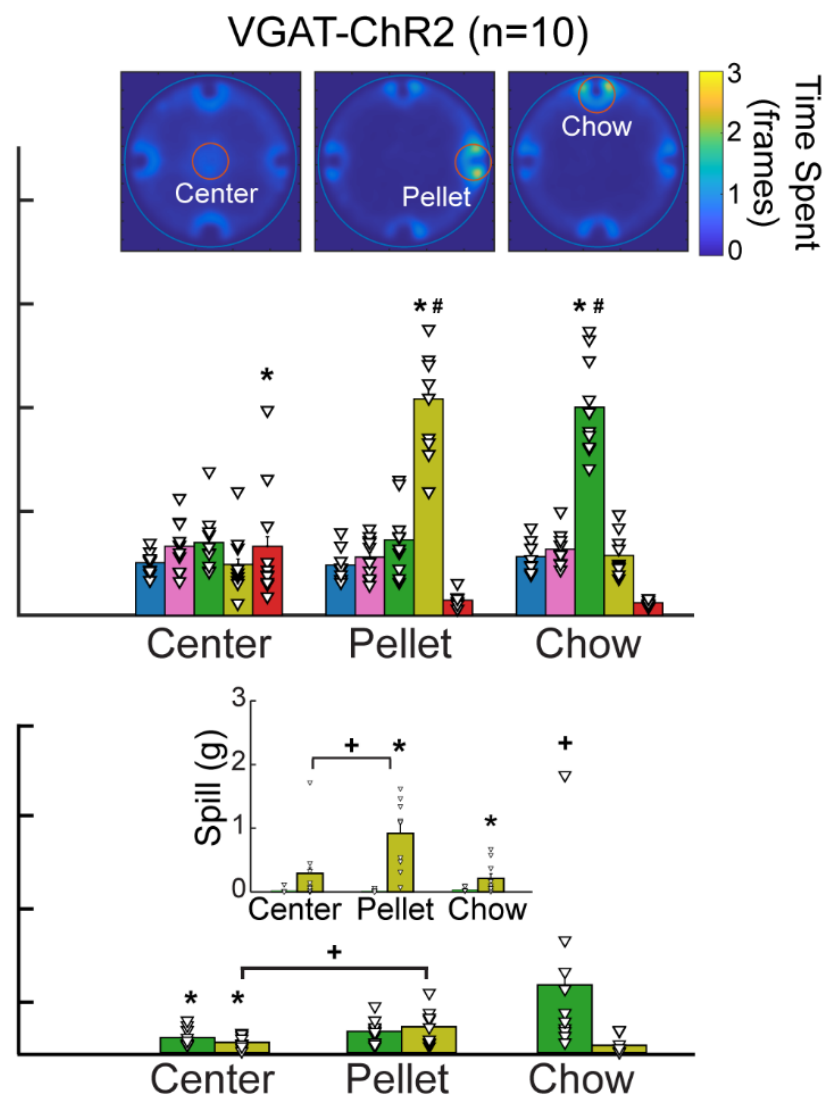

Designated area

Figure 9. Stimulation of LHA ${ }^{\text {Vgat }}$ neurons is rewarding and biases approach towards the nearest stimulus. (A) Different stimuli (plates containing cork, Chow, chocolate pellets, or a sipper filled with 
$118010 \%$ liquid sucrose) were presented simultaneously in a circular arena. In this task, when mice cross a 1181 designated area (dashed circles), the laser was turned on ( $2 \mathrm{~s}$ on; $4 \mathrm{~s}$ off) in a 40 min session. Thus, a 1182 mouse had to leave and re-enter the designated area to receive a new opto-self-stimulation. Only one 1183 designated area was used per session, and it remained in the same position for up to 3 or 4 consecutive 1184 sessions. (B) Heat map and time spent from a representative WT (left panel) and VGAT-ChR2 mice 1185 (right panels), when the designated area was either the center, the pellet, or the Chow (see red circles 1186 for the currently designated zone). Colorbar indicates the number of frames the subject was detected 1187 in a given pixel; higher values indicate it remained in the same place for a longer time. Below, bar 1188 graphs depict the time spent in seconds exploring the designated area (within a radius of $5 \mathrm{~cm}$ ). Each 1189 dot and triangle represent a single individual. Though mice normally avoid exploring the center of an 1190 open field, opto-self-stimulation in the center zone increased the time transgenic mice spent exploring 1191 it, compared with WT. Transgenic mice spent more time exploring the chocolate pellets and the Chow 1192 food when they were in the designated zones. (C) Intake of chocolate pellet and Chow. Activation of 1193 LHA $^{\text {Vgat+ }}$ neurons in the center zone increased chocolate pellets and chow consumption compared with 1194 WT. Inset: Spill from chocolate pellets and chow. * Indicates statistically significant difference $(p<$ 1195 0.05) from WT. $+p<0.05$ shows a significant increase in intake and spill during the session that a 1196 designated area was opto-self-stimulated relative to the sessions where the center was opto-self1197 stimulated. Unpaired Student $t$-test. \# $p<0.01$ indicates a significant difference between designated 1198 area opto-stimulated and the other open field areas. One-way ANOVA followed by the Holm Sidak 1199 test. See Video 5. 


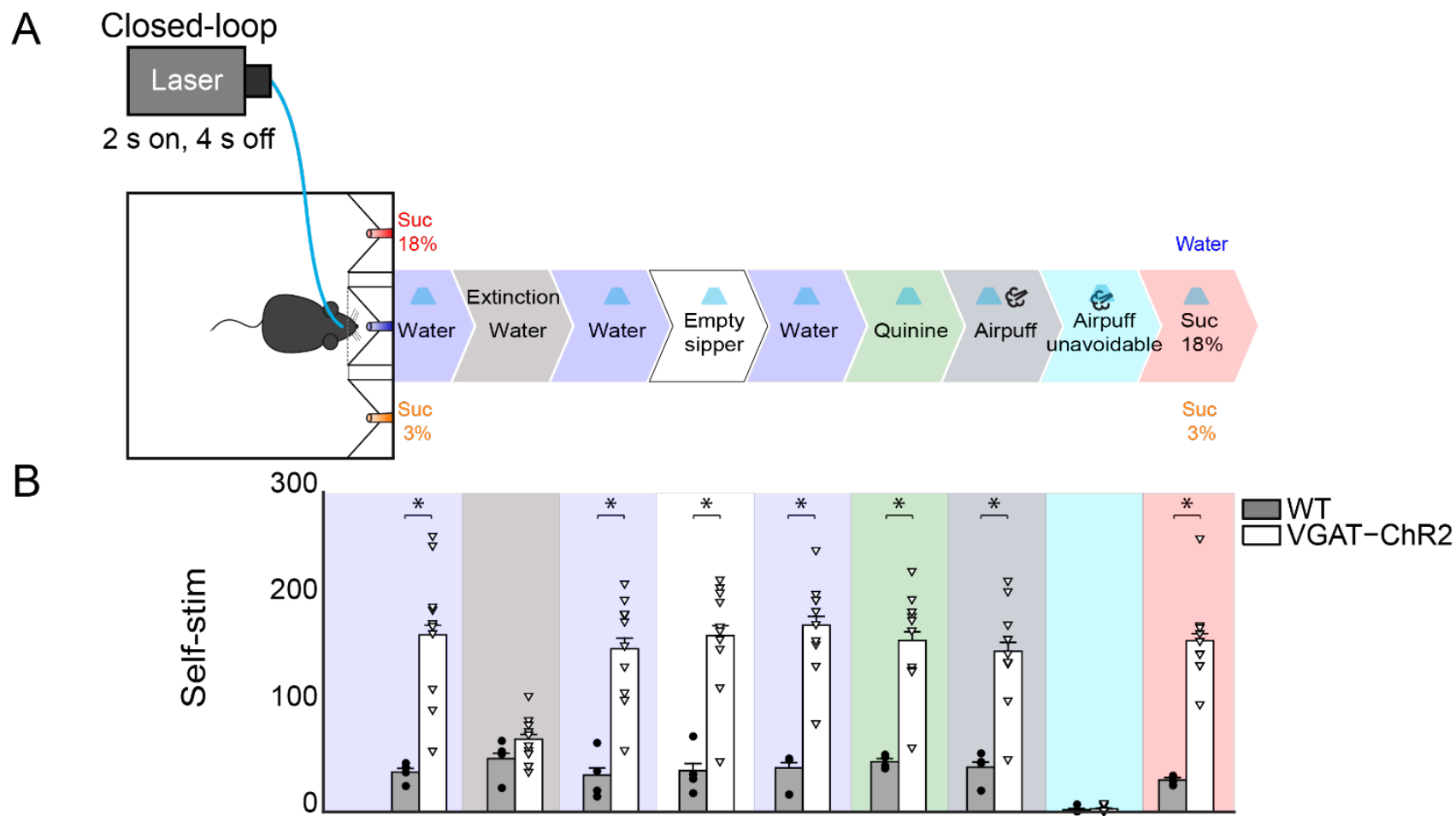

C
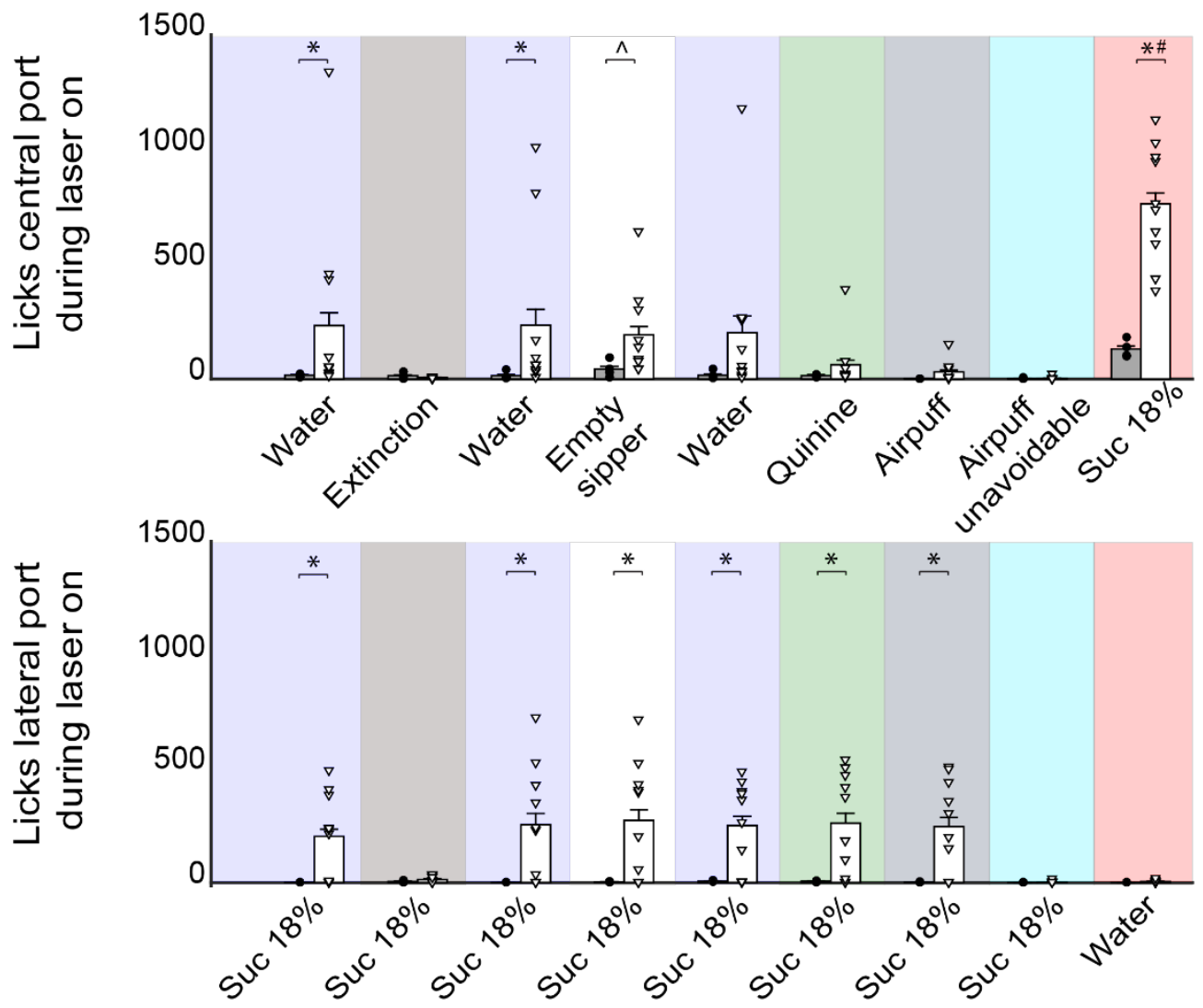

1201 Figure 10. Activating LHA $^{\text {Vgat+ }}$ neurons does not induce the intake of a bitter tastant, nor an unavoidable aversive stimulus, but it increases sucrose consumption. (A) Schematic of behavioral setup showing stimuli delivered at central port. The water stimulus in the central port was replaced by an empty sipper, quinine, or airpuff. In all phases, head-entry in the central port triggered the laser, 
except in the airpuff unavoidable condition, where the first lick delivered both the laser and the airpuff.

Finally, the airpuff in the central port was replaced by sucrose $18 \%$. (B) The number of opto-selfstimulations given for each stimulus. The VGAT-ChR2 mice performed more self-stimulations than WT, except during extinction sessions and when the airpuff was unavoidable. (C) The number of licks given to the central port. The licks in the central port decreased when an aversive stimulus was present, such as quinine or airpuffs. In contrast, a non-edibles stimulus such as an empty sipper elicited more licks from VGAT-ChR2 mice $(p<0.05)$. Moreover, when sucrose $18 \%$ was in the central port, VGATChR2 group increased its consumption substantially compared with WT. (D) The number of licks in the lateral port containing sucrose $18 \%$ during opto-self-stimulation. The intake of the lateral port of sucrose $3 \%$ is not shown because it was neglectable. Each dot and triangle represent a single individual. $\wedge$ Denotes statistically significant difference $(p<0.0001)$ from WT. Unpaired-Student t-test. $* p<0.05$ relative to WT group. \# $p<0.05$ between sucrose $18 \%$ from other stimuli delivered at the central port.
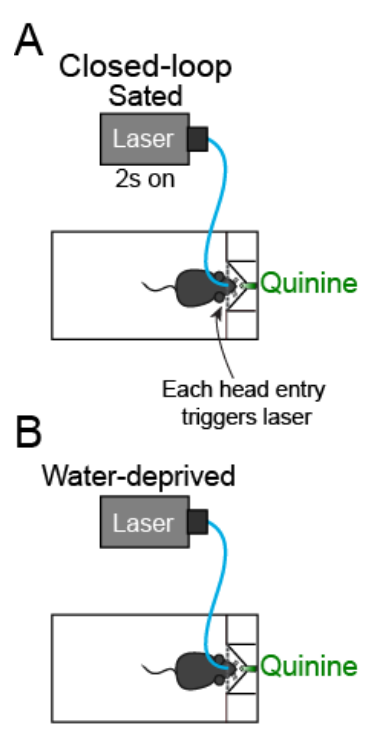

C
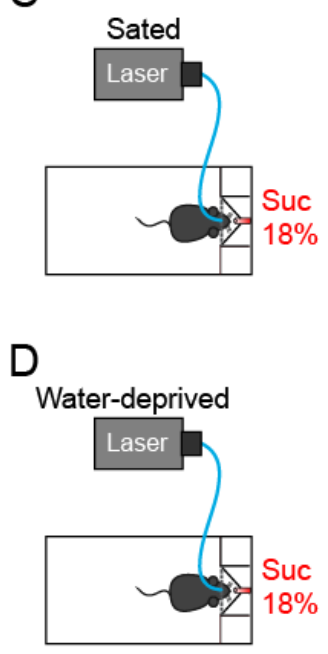
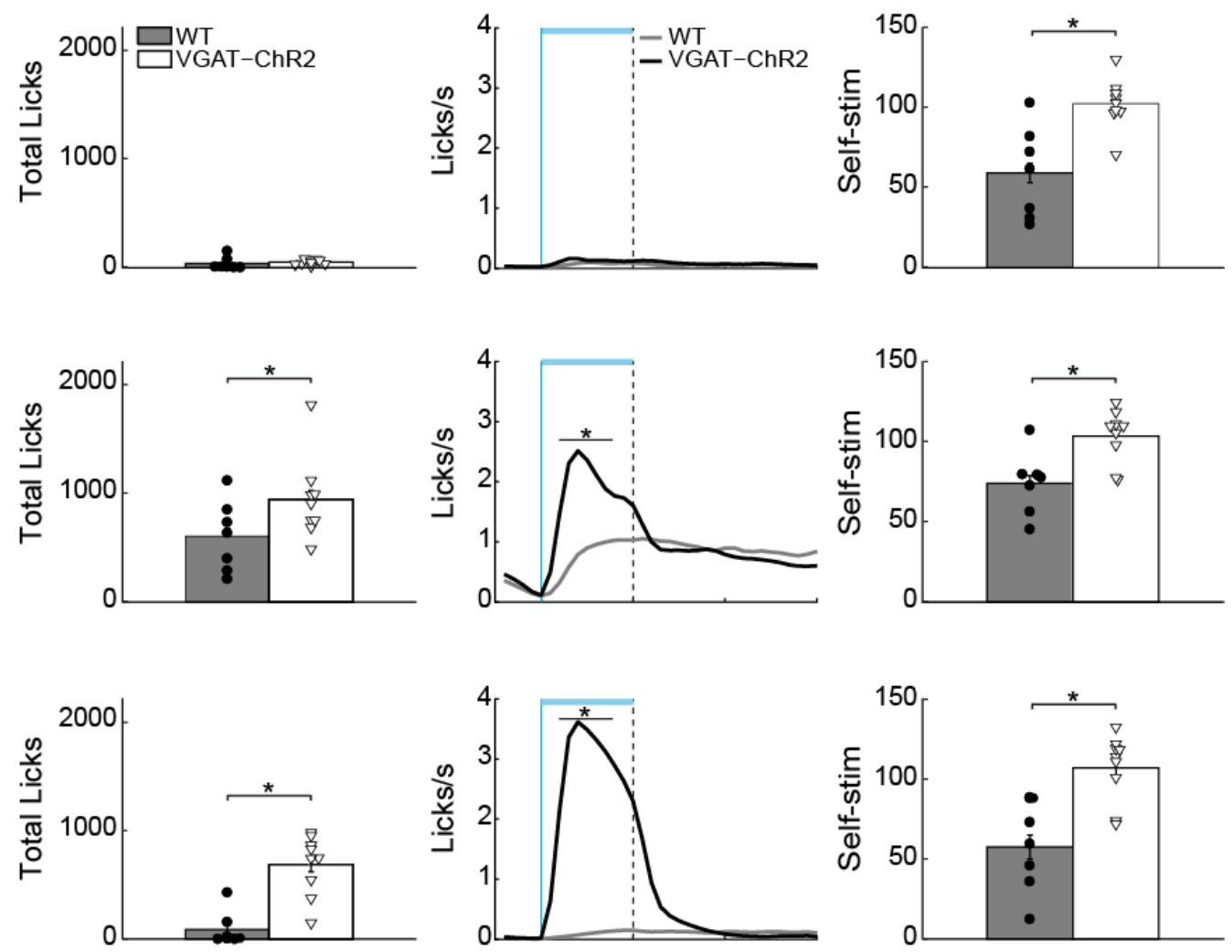

1219
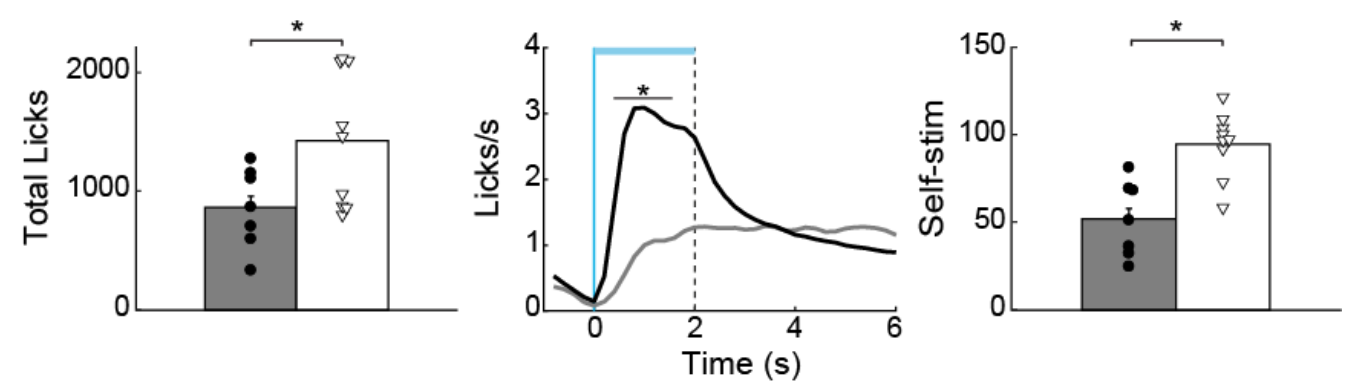
1220 Figure 11. Water deprivation gates a time window where activation of LHA GABAergic neurons increases quinine intake. (A) Sated mice had free access to one sipper filled with quinine. The laser was turned "on" in closed-loop protocol (left panel). Each head entry triggers $2 \mathrm{~s}$ laser "on" followed by a $4 \mathrm{~s}$ time out with no-laser. In sated VGAT-ChR2 mice, activation of these neurons did not increase quinine intake (central panel shows the PSTH of the lick rates aligned to laser onset, time $=0$ s). Although sated transgenic mice do not lick for quinine, they continued opto-self-stimulating (right panel). (B) Water deprived transgenic mice consumed more quinine than WT mice (central panel) when the laser was turned on (horizontal blue line). (C-D) Total licks, PSTH of the lick rate (central panels), and number of opto-self-stimulations of $18 \%$ sucrose during sated and water deprivation condition (right panel). The horizontal blue line indicates opto-self-stimulation window. The vertical blue line indicates the laser onset, and the black dashed line the laser offset. $* p<0.01$ indicates significant differences compared with WT mice according to an unpaired t-student test.
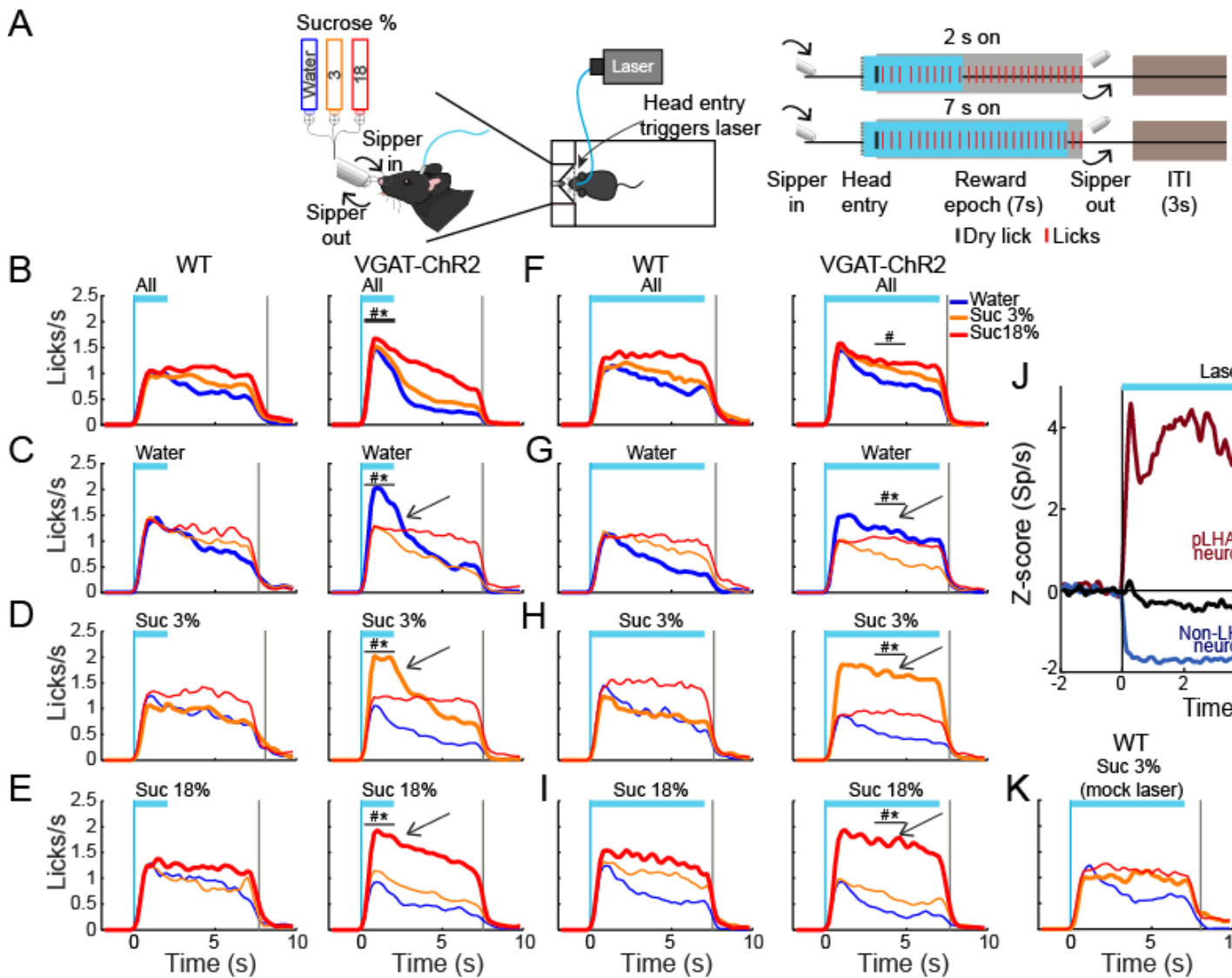

1233
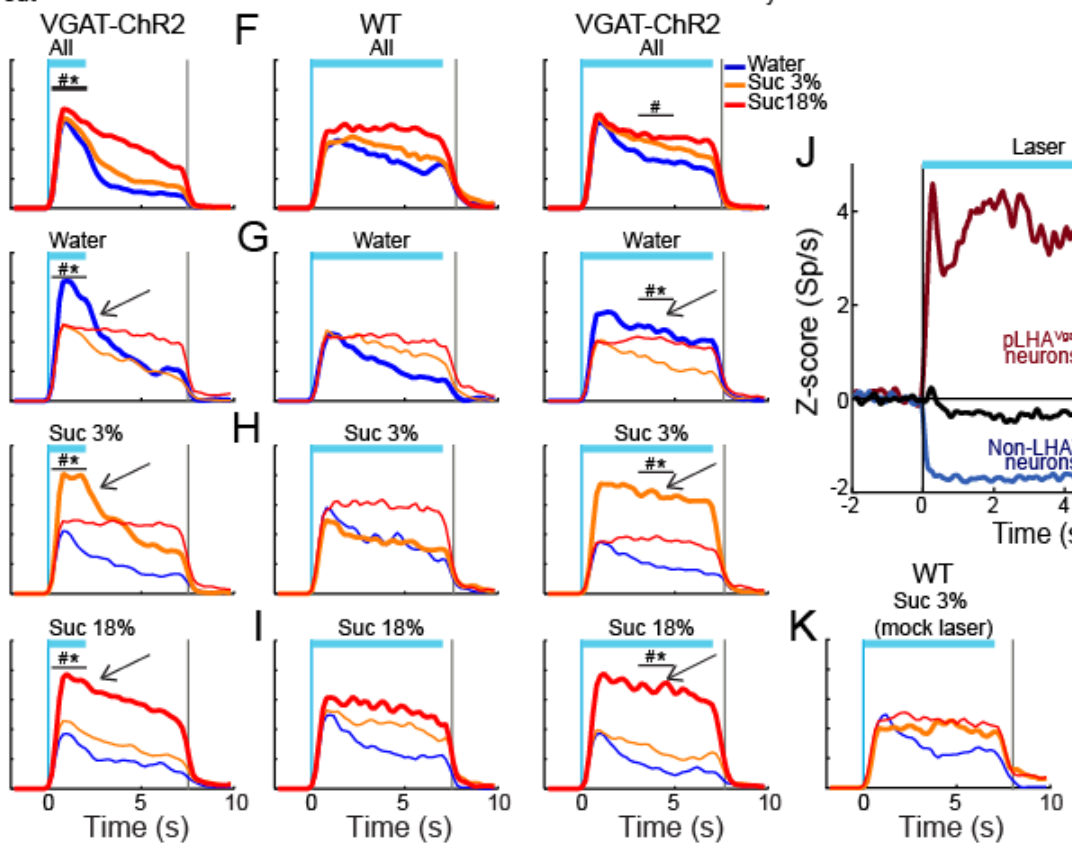

Laser

Figure 12. Activation of LHA GABA neurons enhances water and sucrose's palatability. (A) Closedloop stimulation during the brief access test. Left panel, behavioral setup. The box contained a sipper that delivered a $\sim 2 \mu \mathrm{L}$ drop of either water, sucrose $3 \%$, or $18 \%$ per lick. Right panel, schematics of the structure of a trial. A head entry in the port (dashed vertical line) triggered opto-self-stimulation, at $50 \mathrm{~Hz}$, in some sessions for $2 \mathrm{~s}$ and others for $7 \mathrm{~s}$ (blue rectangles). The reward epoch begins with the first dry lick on the sipper (black tick) and always last 7s (gray rectangles). During the reward epoch, a drop of tastant was delivered in each lick (red marks). At the end of the reward epoch, the sipper was retracted, and an Inter Trial Interval (ITI) of $3 \mathrm{~s}$ began. (B-E) Data of closed-loop for 2s stimulation in the Reward epoch. PSTH of lick rate of WT (left panel) and VGAT-ChR2 mice (right panel) aligned 
1243 (Time $=0 \mathrm{~s}$ ) to laser onset. Opto-self-stimulation was delivered during all trials (B) or during only 1244 water trials (C, see arrow), or sucrose 3\% (D), or $18 \%$ (E). (F-I) Licking responses but during 7 s opto1245 self-stimulation. Same conventions as in B-E. The vertical blue line indicates laser onset and gray line 1246 the end of the reward epoch. The horizontal blue line indicates opto-self-stimulation window. * 1247 Indicates significant difference $(p<0.05)$ from WT group. \# $p<0.05$ among stimuli. Two-way 1248 ANOVA followed by the Holm Sidak test. $\boldsymbol{J}$, Normalized Z-score population activity of 284 neurons 1249 recorded in LHA in VGAT-ChR2 mice $(\mathrm{n}=3)$. The laser was turned on for $7 \mathrm{~s}$ at $50 \mathrm{~Hz}$. Neuronal 1250 responses were aligned to laser onset (Time $=0 \mathrm{~s}$ ). Red and blue colors indicate activated or inhibited 1251 laser-evoked responses, relative to baseline (-1 to $0 \mathrm{~s}$ ). Black trace illustrates neurons unmodulated by 1252 the laser. (K) Mock laser stimulation did not affect palatability responses. PSTH of licking responses, 1253 opto-self-stimulation was delivered along with sucrose 3\% trials during 7s (arrow). Note that both 1254 groups increased the lick rate as a function of palatability, same conventions as in B-E. To elicit mock 1255 laser stimulation, mice were connected to a mock fiber optic (with no-laser connected), whereas the 1256 real fiber was glued outside the mice's head to emit blue light. 